Bull. Soc. math. France

132 (2), 2004, p. 263-304

\title{
RÉSONANCES DE RAYLEIGH EN DIMENSION 2
}

\author{
PAR Didier GAmblin
}

RÉSumÉ. - Nous étudions les résonances de Rayleigh créées par un obstacle strictement convexe à bord analytique en dimension 2. Nous montrons qu'il existe exactement deux suites de résonances $\left(z_{k,+}\right)$ et $\left(z_{k,-}\right)$ convergeant exponentiellement vite vers l'axe réel dans un voisinage polynomial de l'axe réel, et exponentiellement proches d'une suite de quasimodes réels. De plus, $k^{-1} \operatorname{Re} z_{k, \pm}$ est un symbole analytique d'ordre 0 en la variable $k^{-1}$ dont on donne le premier terme du développement. Nous construisons pour cela des quasimodes de Rayleigh dans un voisinage du bord de l'obstacle.

Abstract (Rayleigh Resonances in Two Dimension). - We study the Rayleigh resonances that are created by a strictly convex body with analytic boundary in two dimension. In some polynomial neighbourhood of the real axis we prove that exists exactly two sequences of resonances $\left(z_{k,+}\right)$ and $\left(z_{k,-}\right)$ converging exponentially to the real axis and exponentially close to a sequence of real quasimodes. Moreover, $k^{-1} \operatorname{Re} z_{k, \pm}$ is a zero order analytic symbol in $k^{-1}$ and we give the first term of his expansion. To prove that, we construct Rayleigh quasimodes in a neighbourhood of the obstacle.

Texte reçu le 11 février 2003, accepté le 25 avril 2003

Didier Gamblin, LAGA, Institut Galilée, Université de Paris 13, Avenue Jean-Baptiste Clément, 93430 Villetaneuse • E-mail : gamblin@zeus.math.univ-paris13.fr

Classification mathématique par sujets (2000). - 35P25, 81Q20, 73C02.

Mots clefs. - Ondes de Rayleigh, résonances, construction BKW. 


\section{Introduction, notations et résultats}

Soient $\mathcal{O} \subset \mathbb{R}^{2}$ un compact strictement convexe à bord $\Gamma$ analytique et $\Omega=\mathbb{R}^{2} \backslash \mathcal{O}$ son complémentaire. Notons $\Delta_{e}$ l'opérateur d'élasticité

$$
\Delta_{e} u=\mu_{0} \Delta u+\left(\lambda_{0}+\mu_{0}\right) \nabla(\nabla \cdot u), \quad u={ }^{t}\left(u_{1}, u_{2}\right)
$$

où les constantes de Lamé $\lambda_{0}$ et $\mu_{0}$ vérifient $\mu_{0}>0, \lambda_{0}+\mu_{0}>0$. Considérons $\Delta_{e}$ dans $\Omega$ avec des conditions de Neumann sur le bord $\Gamma$

$$
(B u)_{i}:=\sum_{j=1}^{2} \sigma_{i j}(u) \nu_{j}=0, \quad(i=1,2 \text { sur } \Gamma)
$$

où $\sigma_{i j}(u)=\lambda_{0} \nabla \cdot u \delta_{i j}+\mu_{0}\left(\partial_{x_{j}} u_{i}+\partial_{x_{i}} u_{j}\right)$ est le tenseur des contraintes et $\nu$ la normale extérieure à $\Omega$ sur $\Gamma$.

Pour l'équation des ondes élastiques à l'extérieur d'un obstacle régulier avec condition de Neumann sur le bord M. Taylor [20] a étudié mathématiquement, en dimension quelconque, la propagation des singularités. Trois types de rayons peuvent propager les singularités. D'une part les rayons classiques se réfléchissant au bord de l'obstacle suivant les lois de l'optique géométrique et propageant les singularités aux vitesses $c_{1}=\left(\mu_{0}\right)^{\frac{1}{2}}$ et $c_{2}=\left(\lambda_{0}+2 \mu_{0}\right)^{\frac{1}{2}}$. D'autre part les rayons de Rayleigh propageant les singularités dans le bord de l'obstacle à la vitesse plus lente $c_{R}$. On rappelle que $c_{R}<c_{1}<c_{2}$ et $c_{R}=c_{1} s_{0}$ où $s_{0}$ est l'unique zéro dans ]0, 1[ de la fonction de Rayleigh

$$
\mathcal{R}(s)=\left(s^{2}-2\right)^{2}-4\left(1-s^{2}\right)^{\frac{1}{2}}\left(1-c_{1}^{2} c_{2}^{-2} s^{2}\right)^{\frac{1}{2}} .
$$

Ce qui fait que même un obstacle strictement convexe est captif pour le problème de Neumann du point de vue de la propagation des singularités. Ce qui n'est pas le cas pour le problème de Dirichlet.

M. Ikehata et G. Nakamura [6] dans le cas de la sphère de $\mathbb{R}^{3}$, puis M. Kawashita [7] pour un obstacle à bord $\mathcal{C}^{\infty}$ quelconque en dimension impaire, ont montré que l'énergie locale de l'équation des ondes élastiques pour le problème de Neumann n'a pas la propriété de décroissance uniforme lorsque $t$ tend vers l'infini. Ces phénomènes correspondent à l'existence d'ondes de Rayleigh se propageant au bord de l'obstacle et on s'attendait à ce que cela crée des résonances convergeant rapidement vers l'axe réel.

Il est connu que l'opérateur $-\Delta_{e}$, agissant sur les fonctions $\mathcal{C}^{\infty}$ à support compact dans $\Omega$ à valeurs dans $\mathbb{C}^{2}$ et vérifiant la condition de Neumann, admet une réalisation auto-adjointe sur $L^{2}\left(\Omega ; \mathbb{C}^{2}\right)$ que l'on notera $-\Delta_{e}^{N}$. L'opérateur $-\Delta_{e}^{N}$ est positif et n'admet pas de spectre ponctuel. On considère l'ensemble

$$
\Lambda=\left\{\lambda \in \mathbb{C} ;|\operatorname{Im} \lambda| \leq|\lambda|^{-\delta}, \operatorname{Re} \lambda \geq C_{0}\right\} .
$$

Soit $\chi$ une fonction $\mathcal{C}^{\infty}$ à support compact valant 1 près de $\Gamma$; la résolvante tronquée $R_{\chi}(\lambda)=\chi\left(-\Delta_{e}^{N}-\lambda^{2}\right)^{-1} \chi$, holomorphe pour $\lambda$ dans $\Lambda$ avec $\operatorname{Im} \lambda<0$, se prolonge méromorphiquement à $\Lambda$ tout entier avec des pôles

TOME $132-2004-\mathrm{N}^{\mathrm{O}} 2$ 
possibles pour $\operatorname{Im} \lambda>0$. Ces pôles sont appelés résonances de l'opérateur $-\Delta_{e}^{N}(\operatorname{voir}[11])$.

P. Stefanov et G. Vodev [15] ont commencé par montrer que dans le cas de la boule de $\mathbb{R}^{3}$, il existe une suite de résonances convergeant exponentiellement vite vers l'axe réel. Ils ont ensuite montré (voir [16]) que la convergence était polynomiale, d'abord dans le cas d'un obstacle strictement convexe à bord $\mathcal{C}^{\infty}$ de $\mathbb{R}^{3}$, puis pour un obstacle à bord $\mathcal{C}^{\infty}$ quelconque en dimension impaire d'espace (voir [17]). J. Sjöstrand et G. Vodev [10] ont donné l'asymptotique du nombre de résonances de Rayleigh dans un domaine proche de l'axe réel de la forme

$$
\Lambda:=\left\{\lambda \in \mathbb{C} ;|\operatorname{Im} \lambda| \leq|\lambda|^{-\delta}, \operatorname{Re} \lambda \geq C_{0}\right\}, \quad\left(\delta, C_{0}>0\right),
$$

pour une classe d'obstacles incluant le cas strictement convexe et en toute dimension d'espace autre que 4. P. Stefanov [13] a donné aussi une très bonne minoration du nombre de résonances de Rayleigh pour un obstacle quelconque en dimension 3. G. Vodev [21] a étendu le résultat obtenu dans le cas de la boule de $\mathbb{R}^{3}$ à toute dimension impaire d'espace, en montrant que la convergence est exponentielle dès que l'une des composantes connexes de l'obstacle est à bord analytique. Dans le cas d'un obstacle à bord $\mathcal{C}^{\infty}$ en dimension 3, M. Bellassoued [1] a montré l'existence d'un domaine exponentiellement proche de l'axe réel sans résonance.

Dans le cas d'un obstacle strictement convexe à bord analytique $\Gamma$ en dimension 2, nous obtenons le résultat suivant :

ThÉORÈme 0.1. - Il existe deux suites de résonances $z_{k,+}$ et $z_{k,-}$ de Rayleigh (comptées avec multiplicités) vérifiant

$$
\left|\operatorname{Re} z_{k,+}-\operatorname{Re} z_{k,-}\right|=O\left(\exp \left(-C \operatorname{Re} z_{k, \pm}\right)\right), \quad \operatorname{Im}\left(z_{k, \pm}\right)=O\left(\exp \left(-C \operatorname{Re} z_{k, \pm}\right)\right)
$$

où $C$ est une constante $>0$. Ici $k^{-1} \operatorname{Re} z_{k, \pm}$ est un symbole analytique d'ordre 0 de la variable $k^{-1}$ et

$$
\operatorname{Re}\left(z_{k, \pm}\right)=\frac{2 \pi c_{R}}{\ell(\Gamma)} k+\sum_{m \geq 0} a_{m} k^{-m}
$$

Soit $\Lambda=\left\{z \in \mathbb{C} ;|\operatorname{Im} z| \leq|z|^{-\delta}\right.$, $\left.\operatorname{Re} z \geq C_{0}\right\}$ où $\delta, C_{0}>0$. Si $C_{0}$ est assez grand, il n'y a pas d'autres résonances de Rayleigh dans $\Lambda$ que les nombres $z_{k, \pm}$.

L'intérêt de la localisation précise des résonances réside dans le lien avec le problème d'évolution associé (voir [19] et [14]). Il reste donc à préciser le taux de décroissance exponentielle de la partie imaginaire des résonances en fonction de la géométrie de l'obstacle (l'auteur a travaillé dans cette direction [3]) et à étudier l'intéraction qu'il peut exister entre les résonances $z_{k,+}$ et $z_{k,-}$. 


\section{Autour de la preuve}

L'argument de Stefanov et Vodev était basé sur une estimation a priori du prolongement méromorphe de la résolvante tronquée et une application du principe de Phragmen-Lindelöf. L'existence de quasimodes contredit alors l'absence de résonance près du réel.

En reprenant l'idée de Stefanov et Vodev, mais en appliquant le principe du maximum dans des petits voisinages d'un quasimode, S.-H. Tang et M. Zworski [18] ont montré, pour des perturbations du laplacien et en toutes dimensions d'espace, que près d'un quasimode réel (pourvu qu'il soit assez grand) existait au moins une résonance. Ensuite, Stefanov [12] a étendu les résultats de Tang et Zworski au cas des quasimodes multiples et des clusters de quasimodes. Il montre que près de chaque groupe de quasimodes existent au moins autant de résonances (comptées avec multiplicités) qu'il y a de quasimodes. Cela permet de localiser les résonances dans des boules et pas seulement près de l'axe réel. Nous allons donc construire des quasimodes pour le système de l'élasticité avec une précision exponentiellement petite.

Introduisons l'opérateur de Dirichlet-Neumann $\mathcal{N}(\lambda)$ défini par

$$
\mathcal{N}(\lambda): H^{s}(\Gamma) \ni f \longmapsto B v_{\mid \Gamma} \in H^{s-1}(\Gamma),
$$

où $H^{s}(\Gamma)$ désigne les espaces de Sobolev usuels sur $\Gamma$ et $v$ la solution du problème

$$
\left(\Delta_{e}+\lambda^{2}\right) v=0 \text { dans } \Omega, \quad v=f \text { sur } \Gamma, \quad v \lambda \text {-sortante. }
$$

Rappelons que la fonction $v$ est dite $\lambda$-sortante si pour $r_{0} \gg 1$ nous avons

$$
v_{|| x \mid \geq r_{0}}=R_{0}(\lambda) g_{|| x \mid \geq r_{0}},
$$

où $g$ appartient à $L_{\text {comp }}^{2}(\Omega)$, est à support indépendant de $\lambda$, et $R_{0}(\lambda)$ est la résolvante libre sortante de $-\Delta_{e}$ dans $\mathbb{R}^{2}$; c'est-à-dire que $R_{0}(\lambda)$ appartient à $\mathcal{L}\left(L^{2}(\Omega), L^{2}(\Omega)\right)$ pour $\operatorname{Im} \lambda<0$.

On sait (voir [16] et [21]) que dans la zone elliptique

$$
\mathcal{E}=\left\{\zeta \in T^{*} \Gamma ; c_{1}\|\zeta\|>1\right\},
$$

$\mathcal{N}(\lambda)$ est un opérateur pseudodifférentiel analytique à grand paramètre $\lambda$ ayant pour variété caractéristique

$$
\Sigma=\left\{\zeta \in T^{*} \Gamma ; c_{R}\|\zeta\|=1\right\},
$$

où $c_{R}$ est la vitesse de Rayleigh. C'est l'existence de cette variété caractéristique qui génère les résonances convergeant rapidement vers l'axe réel. Dans le cas de la dimension $2, \Sigma$ est simplement la réunion de deux courbes fermées $\Sigma^{+}$ et $\Sigma^{-}$, ce qui explique la simplicité du problème en dimension 2 par rapport aux dimensions supérieures. On va construire des quasimodes localisés près de $\Sigma^{ \pm}$.

Dans la section 2, puisque $\mathcal{O}$ est strictement convexe, nous définissons des coordonnées d'Euler-Gauss dans $\Omega$, c'est-à-dire que l'une des variables, que l'on

TOME $132-2004-\mathrm{N}^{\mathrm{O}} 2$ 
note $\theta$, est l'angle polaire de la normale au bord $\Gamma$ et l'autre, que l'on note $r$, représente la distance à $\Gamma$. Nous étendons ensuite ces coordonnées dans un voisinage complexe de $\Gamma$. Dans la suite, les variétés sont vues comme des sousvariétés du revêtement universel (via les coordonnées d'Euler-Gauss) des complexifiés de $\Gamma$ ou $\Omega$ ou des fibrés cotangents de ces revêtements.

Dans la section 3, nous construisons quatre lagrangiennes complexes $\Lambda_{j}^{ \pm}$de dimension 2, incluses respectivement dans $p_{j}^{-1}(1)$ (les notations $p_{1}(x, \xi)=c_{1}^{2}|\xi|^{2}$ et $p_{2}(x, \xi)=c_{2}^{2}|\xi|^{2}$ désignent les valeurs propres du symbole principal semiclassique de $-h^{2} \Delta_{e}$ ) et dont la projection sur $T^{*} \Gamma$ est $\Sigma^{ \pm}$. Assez près de $\Gamma$, ces lagrangiennes se projettent régulièrement dans l'espace des configurations.

Dans la section 4 , nous construisons deux solutions BKW $u^{ \pm}$du système

$$
\left(-h^{2} \Delta_{e}-1\right) u=0 \text { près de } \Gamma, \quad B u=0 \text { sur } \Gamma,
$$

qui décroissent exponentiellement près du bord de l'obstacle (ici $\Gamma$ est en fait remplaçé par son revêtement universel). La solution BKW $u^{ \pm}$que l'on obtient vit à la fois sur les deux lagrangiennes $\Lambda_{1}^{ \pm}$et $\Lambda_{2}^{ \pm}$. Elle est de la forme

$$
u^{ \pm}(r, \theta ; h)=u^{1, \pm}(r, \theta ; h) \exp \left(i \phi_{1}^{ \pm}(r, \theta) / h\right)+u^{2, \pm}(r, \theta ; h) \exp \left(i \phi_{2}^{ \pm}(r, \theta) / h\right)
$$

où $u^{j, \pm}(r, \theta ; h)$ est un symbole analytique, ce qui permet après ressommation d'obtenir des erreurs exponentiellement petites par rapport à $h$.

Dans la section 5, nous obtenons une condition de quantification comme condition de recollement de la solution $u^{ \pm}$. Nous montrons que la condition de quantification, indépendante de \pm , est vérifiée pour un symbole analytique réel $h_{k}$ d'ordre -1 de la variable $k^{-1}\left(k \in \mathbb{N}^{*}\right)$ dont le premier terme du développement est $\ell(\Gamma) /\left(2 \pi c_{R}\right) k^{-1}$. Pour cela nous démontrons des résultats sur la composition par rapport à la variable $h$ des symboles analytiques ne dépendant que de $h$.

Dans la section 6 , nous montrons qu'il existe deux résonances $z_{k,+}$ et $z_{k,-}$ (comptées avec multiplicités) exponentiellement proches (en la variable $k$ ) de chaque nombre $1 / h_{k}$ et que dans un domaine de la forme

$$
\Lambda:=\left\{\lambda \in \mathbb{C} ;|\operatorname{Im} \lambda| \leq|\lambda|^{-\delta}, \operatorname{Re} \lambda \geq C_{0}\right\},
$$

il n'y a pas d'autres résonances que celles-là. D'après la section 5 , les parties réelles des résonances sont donc des symboles analytiques d'ordre 1 et le premier terme de leur développement est $2 \pi c_{R} / \ell(\Gamma) k$.

Pour cela, à l'aide des solutions BKW $u^{ \pm}$, nous construisons, pour $h$ appartenant à $\left\{h_{k} ; k \in \mathbb{N}^{*}\right\}$, des fonctions $W^{ \pm}(h)$ à support compact dans $\Omega$, de norme 1 dans $L^{2}(\Omega)$ et vérifiant

$$
\begin{cases}\left(-h^{2} \Delta_{e}-1\right) W^{ \pm}(h)=O(\exp (-C / h)) & \text { dans } \Omega \\ \left(W^{+}(h) \mid W^{-}(h)\right)_{L^{2}(\Omega)}=O(\exp (-C / h)), & \\ B W^{ \pm}(h)=0 & \text { sur } \Gamma\end{cases}
$$

BULLETIN DE LA SOCIÉtÉ MATHÉMATIQUE DE FRANCE 
Les résultats de Stefanov [12] nous donnent alors l'existence des deux résonances (comptées avec multiplicités) exponentiellement proches de $h_{k}{ }^{-1}$, et l'asymptotique du nombre de résonances de Rayleigh trouvée par Sjöstrand et Vodev [10], dont le reste est très bon, permet d'affirmer qu'il n'y en a pas d'autres.

\section{Coordonnées d'Euler-Gauss}

2.1. Dans $\Omega$. - La courbe $\Gamma$ peut être vue comme l'enveloppe des droites d'équation $x_{1} \cos \theta+x_{2} \sin \theta=p(\theta)$ où $p$ est analytique et $2 \pi$ périodique. Elle a pour représentation paramétrique

$$
\begin{aligned}
& x_{1_{\Gamma}}(\theta)=p(\theta) \cos \theta-p^{\prime}(\theta) \sin \theta, \\
& x_{2_{\Gamma}}(\theta)=p(\theta) \sin \theta+p^{\prime}(\theta) \cos \theta .
\end{aligned}
$$

Le rayon de courbure de $\Gamma$ au point $\left(x_{1_{\Gamma}}(\theta), x_{2_{\Gamma}}(\theta)\right)$ est $R(\theta)=p(\theta)+p^{\prime \prime}(\theta)$. Le compact $\mathcal{O}$ étant strictement convexe, on a $R(\theta)>0$.

La longueur de $\Gamma$ vaut $\ell(\Gamma)=\int_{0}^{2 \pi}\left(\left(x_{1_{\Gamma}}^{\prime}(\theta)\right)^{2}+\left(x_{2_{\Gamma}}^{\prime}(\theta)\right)^{2}\right)^{\frac{1}{2}} \mathrm{~d} \theta=\int_{0}^{2 \pi} R(\theta) \mathrm{d} \theta$.

La courbe $\Gamma$ est un cercle de rayon $R$ si et seulement si $p(\theta)+p^{\prime \prime}(\theta)=R$, c'est-à-dire si $p(\theta)=R+\alpha \cos \theta+\beta \sin \theta$ (le centre de $\Gamma$ est alors $(\alpha, \beta)$.)

Soit l'application

$$
f:(r, \theta) \longmapsto\left(x_{1_{\Gamma}}(\theta), x_{2_{\Gamma}}(\theta)\right)+r(\cos \theta, \sin \theta) .
$$

Le compact $\mathcal{O}$ étant strictement convexe, pour tout $\left(x_{1}, x_{2}\right)$ dans $\Omega$, il existe $(r, \theta)$ dans $] 0,+\infty[\times \mathbb{R}$ tel que

$$
f(r, \theta)=\left(x_{1}, x_{2}\right) .
$$

$r=d\left(\left(x_{1}, x_{2}\right), \Gamma\right)$ et $\theta$ est unique modulo $2 \pi$. Puisque

$$
\mathrm{d} f=\left(\begin{array}{cc}
\cos \theta-(r+R(\theta)) \sin \theta \\
\sin \theta & (r+R(\theta)) \cos \theta
\end{array}\right), \quad \operatorname{Jac}(f)=r+R(\theta), \quad R(\theta)>0,
$$

on a $\operatorname{Jac}(f) \neq 0$ et $f$ est un difféomorphisme local. En fait $f$ est un revêtement universel. On a

$$
{ }^{t}(\mathrm{~d} f)^{-1}=\frac{1}{r+R(\theta)}\left(\begin{array}{cc}
(r+R(\theta)) \cos \theta & -\sin \theta \\
(r+R(\theta)) \sin \theta & \cos \theta
\end{array}\right) .
$$

Donc

$$
\partial_{x_{1}}=\cos \theta \partial_{r}-\frac{\sin \theta}{r+R(\theta)} \partial_{\theta} \quad \text { et } \quad \partial_{x_{2}}=\sin \theta \partial_{r}+\frac{\cos \theta}{r+R(\theta)} \partial_{\theta} .
$$

Si $\left(\xi_{1}, \xi_{2}\right)$ sont les coordonnées duales de $\left(x_{1}, x_{2}\right)$, le symbole matriciel de $-\Delta_{e}$ est $\mu_{0}|\xi|^{2} I+\left(\lambda_{0}+\mu_{0}\right)\left(\xi_{i} \xi_{j}\right)_{1 \leq i \leq 2,1 \leq j \leq 2}$ et a pour valeurs propres

$$
p_{1}(x, \xi)=c_{1}^{2}|\xi|^{2} \quad \text { et } \quad p_{2}(x, \xi)=c_{2}^{2}|\xi|^{2} \text {. }
$$

TOME $132-2004-\mathrm{N}^{\mathrm{O}} 2$ 
Si $(\rho, \eta)$ sont les coordonnées duales de $(r, \theta)$, les valeurs propres du symbole principal matriciel de $-\Delta_{e}$ dans les coordonnées d'Euler-Gauss sont alors

$$
c_{1}^{2}\left(\rho^{2}+\eta^{2} /(r+R(\theta))^{2}\right) \quad \text { et } \quad c_{2}^{2}\left(\rho^{2}+\eta^{2} /(r+R(\theta))^{2}\right) .
$$

Dans les coordonnées $(r, \theta)$, les conditions de Neumann sur le bord $\Gamma$ s'écrivent

$$
B u_{\mid \Gamma}=0 \quad \text { où } \quad B=B_{0}(\theta) \partial_{r}+B_{1}(\theta) \partial_{\theta}
$$

avec

$$
\begin{aligned}
& B_{0}(\theta)=-\left(\begin{array}{cc}
\left(\lambda_{0}+\mu_{0}\right) \cos ^{2} \theta+\mu_{0} & \frac{1}{2}\left(\lambda_{0}+\mu_{0}\right) \sin 2 \theta \\
\frac{1}{2}\left(\lambda_{0}+\mu_{0}\right) \sin 2 \theta & \left(\lambda_{0}+\mu_{0}\right) \sin ^{2} \theta+\mu_{0}
\end{array}\right), \\
& B_{1}(\theta)=\frac{1}{R(\theta)}\left(\begin{array}{cc}
\frac{1}{2}\left(\lambda_{0}+\mu_{0}\right) \sin 2 \theta & -\lambda_{0} \cos ^{2} \theta+\mu_{0} \sin ^{2} \theta \\
-\mu_{0} \cos ^{2} \theta+\lambda_{0} \sin ^{2} \theta & -\frac{1}{2}\left(\lambda_{0}+\mu_{0}\right) \sin 2 \theta
\end{array}\right) .
\end{aligned}
$$

2.2. Dans le complexe. - La courbe $\Gamma$ étant analytique, il existe $a>0$ tel que $p(\theta)$ et $R(\theta)$ sont holomorphes dans la bande complexe

$$
B_{a}=\{\theta \in \mathbb{C} ;|\operatorname{Im}(\theta)|<a\} .
$$

Proposition 2.1. - Si $r_{0}>0$ et a $>0$ sont assez petits, alors l'application

$$
\begin{gathered}
f: A:=\left\{r \in \mathbb{C} ;|r|<r_{0}\right\} \times B_{a} \longrightarrow f(A), \\
(r, \theta) \longmapsto\left(x_{1_{\Gamma}}(\theta), x_{2_{\Gamma}}(\theta)\right)+r(\cos \theta, \sin \theta)
\end{gathered}
$$

est un revêtement universel. Plus précisément, pour $\left(x_{1}, x_{2}\right) \in f(A)$, il existe une seule solution dans $A$ modulo $(0,2 \pi)$ de l'équation $f(r, \theta)=\left(x_{1}, x_{2}\right)$.

Démonstration. - On a $\operatorname{Jac}(f)=r+R(\theta)$ et $R(\theta)>0$ pour $\theta$ réel; donc si $r_{0}$ et $a$ sont assez petits, $\operatorname{Jac}(f) \neq 0$ et $f$ est un difféomorphisme holomorphe local. De plus, $f(r, \theta+2 \pi)=f(r, \theta)$ et $f$ vérifie la dernière propriété de la proposition $\operatorname{sur}\{0\} \times \mathbb{R}$; donc si $r_{0}$ et $a$ sont assez petits, c'est aussi le cas sur $A$.

\section{Constructions géométriques}

3.1. Construction de $\mathbb{C}$-lagrangiennes. - Nous allons construire quatre $\mathbb{C}$-lagrangiennes $\Lambda_{j}^{ \pm}$de dimension 2 incluses respectivement dans $p_{j}^{-1}(1)$ et dont la projection sur $T^{*} \Gamma$ est $\Sigma^{ \pm}$(ici $\Gamma$ et $\Sigma^{ \pm}$désignent les complexifiés de leurs versions réelles). Localement dans les coordonnées $(\theta, \eta), \Sigma^{ \pm}$a pour équation $\eta= \pm c_{R}^{-1} R(\theta)$ (voir section 4). On considère

$$
\begin{aligned}
\Lambda_{j}^{ \pm 0}:=\left\{\left(x_{1}, x_{2}, \xi_{1}, \xi_{2}\right)\right. & \in T^{*} \mathbb{C}^{2} ; r=0, \theta \in B_{a}, \\
& \left.\rho=i\left(c_{R}^{-2}-c_{j}^{-2}\right)^{\frac{1}{2}}, \eta= \pm c_{R}^{-1} R(\theta)\right\} .
\end{aligned}
$$

BULLETIN DE LA SOCIÉTÉ MATHÉMATIQUE DE FRANCE 
Soit la 1-forme canonique $\omega=\xi_{1} \mathrm{~d} x_{1}+\xi_{2} \mathrm{~d} x_{2}$; dans les coordonnées $(r, \theta, \rho, \eta)$, $\omega=\rho \mathrm{d} r+\eta \mathrm{d} \theta$. Si l'on pose $\sigma=\mathrm{d} \omega$, on a $\sigma_{\mid \Lambda_{j}^{ \pm 0}}=0$ et $\Lambda_{j}^{ \pm 0}$ est une sous-variété de dimension 1 sur $\mathbb{C}$ incluse dans l'hypersurface $p_{j}^{-1}(1)$.

$H_{p_{j}}=2 c_{j}^{2} \xi \cdot \partial_{x}$ ne s'annule pas sur $p_{j}^{-1}(1)$. On définit donc, pour $T>0$

$$
\Lambda_{j}^{ \pm}:=\left\{\exp \left(t H_{p_{j}}\right)(\nu) ; \nu \in \Lambda_{j}^{ \pm 0}, t \in \mathbb{C} \text { et }|t|<T\right\} .
$$

$H_{p_{j}}$ est tangent à $p_{j}^{-1}(1)$, donc on a $\Lambda_{j}^{ \pm} \subset p_{j}^{-1}(1)$; de plus $H_{p_{j}}$ est transverse à $\Lambda_{j}^{ \pm 0}$, donc la dimension de $\Lambda_{j}^{ \pm}$est 2 et $\sigma_{\mid \Lambda_{j}^{ \pm}}=0$, donc $\Lambda_{j}^{ \pm}$est une $\mathbb{C}$ lagrangienne. Voir par exemple [4] où cela est fait dans le cas réel.

Les courbes de $H_{p_{j}}$ sont les droites d'équations

$$
\begin{array}{ll}
x_{1}(t)=x_{1}(0)+2 c_{j}^{2} \xi_{1}(0) t, & x_{2}(t)=x_{2}(0)+2 c_{j}^{2} \xi_{2}(0) t, \\
\xi_{1}(t)=\xi_{1}(0), & \xi_{2}(t)=\xi_{2}(0) .
\end{array}
$$

$\Lambda_{j}^{ \pm}$est donc une famille de droites passant par les points de $\Lambda_{j}^{ \pm 0}$ et paramétrée par $\theta$; puisque

$$
\xi_{1}=\rho \cos \theta-\frac{\sin \theta}{r+R(\theta)} \eta \quad \text { et } \quad \xi_{2}=\rho \sin \theta+\frac{\cos \theta}{r+R(\theta)} \eta,
$$

ces droites ont pour équations :

$$
\begin{aligned}
& x_{1}(t)=p(\theta) \cos \theta-p^{\prime}(\theta) \sin \theta+2 c_{j}^{2} t\left(i \cos \theta\left(c_{R}^{-2}-c_{j}^{-2}\right)^{\frac{1}{2}} \mp c_{R}^{-1} \sin \theta\right), \\
& x_{2}(t)=p(\theta) \sin \theta+p^{\prime}(\theta) \cos \theta+2 c_{j}^{2} t\left(i \sin \theta\left(c_{R}^{-2}-c_{j}^{-2}\right)^{\frac{1}{2}} \pm c_{R}^{-1} \cos \theta\right), \\
& \xi_{1}(t)=i \cos \theta\left(c_{R}^{-2}-c_{j}^{-2}\right)^{\frac{1}{2}} \mp c_{R}^{-1} \sin \theta, \\
& \xi_{2}(t)=i \sin \theta\left(c_{R}^{-2}-c_{j}^{-2}\right)^{\frac{1}{2}} \pm c_{R}^{-1} \cos \theta .
\end{aligned}
$$

Pour faciliter l'écriture, on considère les nombres strictement positifs $a_{1}$ et $a_{2}$ $\left(a_{1}<a_{2}\right)$ définis par

$$
\cosh \left(a_{j}\right)=c_{j} c_{R}^{-1} \quad \text { et } \quad \sinh \left(a_{j}\right)=c_{j}\left(c_{R}^{-2}-c_{j}^{-2}\right)^{\frac{1}{2}}, \quad j=1,2 .
$$

Les courbes ont alors pour équations :

$$
\begin{aligned}
& x_{1}(t)=p(\theta) \cos \theta-p^{\prime}(\theta) \sin \theta \mp 2 c_{j} t \sin \left(\theta \mp i a_{j}\right), \\
& x_{2}(t)=p(\theta) \sin \theta+p^{\prime}(\theta) \cos \theta \pm 2 c_{j} t \cos \left(\theta \mp i a_{j}\right), \\
& \xi_{1}(t)=\mp c_{j}^{-1} \sin \left(\theta \mp i a_{j}\right), \\
& \xi_{2}(t)= \pm c_{j}^{-1} \cos \left(\theta \mp i a_{j}\right) .
\end{aligned}
$$

$\Lambda_{j}^{ \pm}$est paramétrée par $(\theta, t)$ où $t$ est unique et $\theta$ est unique modulo $2 \pi$.

TOME $132-2004-\mathrm{N}^{\mathrm{O}} 2$ 
Nous allons voir si $\Lambda_{j}^{ \pm}$se projette bien dans l'espace des configurations. $\mathrm{Au}$ voisinage de tout point de $\Lambda_{j}^{ \pm}, \Pi_{\mid \Lambda_{j}^{ \pm}}:(\theta, t) \mapsto\left(x_{1}, x_{2}\right)$ a pour jacobien

$$
\begin{array}{r}
\left|\begin{array}{rr}
-R(\theta) \sin \theta \mp 2 c_{j} t \cos \left(\theta \mp i a_{j}\right) & \mp 2 c_{j} \sin \left(\theta \mp i a_{j}\right) \\
+R(\theta) \cos \theta \mp 2 c_{j} t \sin \left(\theta \mp i a_{j}\right) & \pm 2 c_{j} \cos \left(\theta \mp i a_{j}\right)
\end{array}\right| \\
=-4 c_{j}^{2}\left(t+\frac{i}{2} c_{j}^{-1} \sinh \left(a_{j}\right) R(\theta)\right) .
\end{array}
$$

Il s'ensuit que le jacobien de $\Pi_{\mid \Lambda_{j}^{ \pm}}$est nul pour $t=-\frac{1}{2} i c_{j}^{-1} \sinh \left(a_{j}\right) R(\theta)$. Si $a$ et $T$ sont assez petits, l'équation n'a pas de solution dans $\Lambda_{j}^{ \pm}$. On en déduit que $\Pi_{\mid \Lambda_{j}^{ \pm}}$est un difféomorphisme holomorphe local. De plus $\Pi_{\mid \Lambda_{j}^{ \pm 0}}$ est injective et $\Pi\left(\Lambda_{j}^{ \pm 0}\right)=\Gamma$ (complexifié) ; on obtient donc la proposition :

Proposition 3.1. - Si a et $T$ sont assez petits, $\Pi_{\mid \Lambda_{j}^{ \pm}}$est bijective de $\Lambda_{j}^{ \pm}$ sur $\Pi\left(\Lambda_{j}^{ \pm}\right)$et $\Pi\left(\Lambda_{j}^{ \pm}\right)$contient un voisinage du complexifié de $\Gamma$.

\subsection{Relèvement des lagrangiennes et construction des phases $\phi_{j}^{ \pm}$}

On va relever les lagrangiennes $\Lambda_{j}^{ \pm}$(via les coordonnées d'Euler-Gauss) afin que la projection dans l'espace des configurations de ces lagrangiennes soit un ouvert contractile.

Précisons le lien entre les coordonnées euclidiennes et celles d'Euler-Gauss dans le cotangent. On considère l'application

$$
g:(r, \alpha, \rho, \eta) \longmapsto\left[f(r, \alpha),^{t}(\mathrm{~d} f)_{(r, \alpha)}^{-1}(\rho, \eta)\right] .
$$

D'après la proposition 2.1, on obtient que $g$ est un difféomorphisme holomorphe local sur $T^{*} A$ et que tout $\left(x_{1}, x_{2}, \xi_{1}, \xi_{2}\right)$ appartenant à $g\left(T^{*} A\right)$ admet un unique antécédent $(r, \alpha, \rho, \eta)$ (modulo $(0,2 \pi, 0,0))$ par $g$ appartenant à $T^{*} A$.

Ceci nous permet de préciser le lien qu'il y a entre les courbes de $H_{p_{j}(r, \alpha, \rho, \eta)}$ et celles de $H_{p_{j}\left(x_{1}, x_{2}, \xi_{1}, \xi_{2}\right)}$. Remarquons d'abord que pour tout $\nu$, on a

$$
\exp \left(t H_{p_{j}}\right)(\nu+(0,2 \pi, 0,0))=\exp \left(t H_{p_{j}}\right)(\nu)+(0,2 \pi, 0,0) .
$$

Les courbes de $H_{p_{j}(r, \alpha, \rho, \eta)}$ relèvent les courbes de $H_{p_{j}\left(x_{1}, x_{2}, \xi_{1}, \xi_{2}\right)}$; plus précisément, si $\gamma$ est une courbe de $H_{p_{j}(r, \alpha, \rho, \eta)}$, alors $g(\gamma)$ est une courbe de $H_{p_{j}\left(x_{1}, x_{2}, \xi_{1}, \xi_{2}\right)}$ et si $\Upsilon$ est une courbe de $H_{p_{j}\left(x_{1}, x_{2}, \xi_{1}, \xi_{2}\right)}$, alors $g^{-1}(\Upsilon)$ est une réunion de courbes disjointes de $H_{p_{j}(r, \alpha, \rho, \eta)}$ et $g^{-1}(\Upsilon) \simeq \gamma \times \mathbb{Z}$, où $\gamma$ est une courbe de $H_{p_{j}(r, \alpha, \rho, \eta)}$.

On va maintenant relever par $g$ les lagrangiennes $\Lambda_{j}^{ \pm}$.

Posons $\Lambda_{j, r}^{ \pm}=g^{-1}\left(\Lambda_{j}^{ \pm}\right)$. D'après la proposition 3.1, la restriction de la projection canonique $\Pi$ à $\Lambda_{j, r}^{ \pm}$réalise un difféomorphisme de $\Lambda_{j, r}^{ \pm} \operatorname{sur} \Pi\left(\Lambda_{j, r}^{ \pm}\right):=\Omega_{j}^{ \pm}$. Il existe donc des fonctions $\psi_{j}^{ \pm}$et $\Phi_{j}^{ \pm}$holomorphes sur $\Omega_{j}^{ \pm}$telles que

$$
\Lambda_{j, r}^{ \pm}=\left\{\left(r, \alpha, \psi_{j}^{ \pm}(r, \alpha), \Phi_{j}^{ \pm}(r, \alpha)\right) ;(r, \alpha) \in \Omega_{j}^{ \pm}\right\} .
$$

BULlETiN DE LA SOCIÉtÉ MATHÉMATIQUE DE FRANCE 
Comme $(\theta, t)$ est un système de coordonnées globales de $\Lambda_{j, r}^{ \pm}$et $A$ est contractile, alors $\Omega_{j}^{ \pm}$est contractile. Comme $\Lambda_{j, r}^{ \pm}$est une lagrangienne, il existe des fonctions $\phi_{j}^{ \pm}$holomorphes sur $\Omega_{j}^{ \pm}$telles que

$$
\Lambda_{j, r}^{ \pm}=\left\{\left(r, \alpha, \partial_{r} \phi_{j}^{ \pm}(r, \alpha), \partial_{\alpha} \phi_{j}^{ \pm}(r, \alpha)\right) ;(r, \alpha) \in \Omega_{j}^{ \pm}\right\} .
$$

De plus, $f\left(\Omega_{j}^{ \pm}\right)$contient un voisinage du complexifié de $\Gamma$ d'après la proposition 3.1. Puisque $\Lambda_{j, r}^{ \pm} \subset p_{j}^{-1}(1)$, on obtient la première partie de la proposition suivante :

Proposition 3.2. - Il existe des fonctions $\phi_{j}^{ \pm}, j=1,2$, holomorphes dans un domaine $\Omega_{j}^{ \pm}$tel que $f\left(\Omega_{j}^{ \pm}\right)$contienne un voisinage du complexifié de $\Gamma$, et qui vérifient

$$
\begin{aligned}
& c_{j}^{2}\left[\left(\partial_{r} \phi_{j}^{ \pm}\right)^{2}+\frac{1}{(r+R(\theta))^{2}}\left(\partial_{\theta} \phi_{j}^{ \pm}\right)^{2}\right]=1, \\
& \partial_{r} \phi_{j}^{ \pm}(0, \theta)=i\left(c_{R}^{-2}-c_{j}^{-2}\right)^{\frac{1}{2}} \quad \text { et } \partial_{\theta} \phi_{j}^{ \pm}(0, \theta)= \pm c_{R}^{-1} R(\theta) .
\end{aligned}
$$

Les fonctions $\phi_{j}^{ \pm}$vérifiant ces trois conditions sont entièrement déterminées à une constante près. De plus, pour $j=1,2$ on a

$$
\phi_{j}^{ \pm}(r, \theta+2 \pi)-\phi_{j}^{ \pm}(r, \theta)= \pm c_{R}^{-1} \ell(\Gamma),
$$

et donc $\operatorname{Im} \phi_{j}^{ \pm}$est $2 \pi$-périodique en la variable $\theta$.

Fin de la preuve. - La condition (2) détermine $\phi_{j}^{ \pm}(0, \theta)$ à une constante près. La fonction $\phi_{j}^{ \pm}(0, \theta)$ vérifiant (2) étant donnée, si $\phi_{j}^{ \pm}$vérifie (1), sa valeur est imposée sur les projections des courbes du champ hamiltonien $H_{p_{j}}$ issues de $\Lambda_{j}^{ \pm 0}$. D'où l'unicité à une constante près de $\phi_{j}^{ \pm}$vérifiant (1) et (2).

Les fonctions $\partial_{r} \phi_{j}^{ \pm}$et $\partial_{\theta} \phi_{j}^{ \pm}$étant $2 \pi$-périodiques en la variable $\theta$, on en déduit les égalités

$$
\begin{aligned}
\phi_{j}^{ \pm}(r, \theta+2 \pi)-\phi_{j}^{ \pm}(r, \theta) & =\phi_{j}^{ \pm}(0, \theta+2 \pi)-\phi_{j}^{ \pm}(0, \theta) \\
& =\int_{0}^{2 \pi} \partial_{\theta} \phi_{j}^{ \pm}(0, \theta) \mathrm{d} \theta= \pm c_{R}^{-1} \ell(\Gamma)
\end{aligned}
$$

On obtient la fin de la proposition en prenant la partie imaginaire.

\section{Construction BKW complexe}

4.1. Introduction. - On cherche à construire des solutions approchées du problème suivant :

$$
Q(h) u=0 \text { près de } \Gamma, \quad B u=0 \text { sur } \Gamma
$$

où $Q(h)$ est l'opérateur $-h^{2} \Delta_{e}-1$ dans les coordonnées $(r, \theta)$ et où et $\Gamma$ est remplaçé par son revêtement universel (via les coordonnées d'Euler-Gauss).

TOME $132-2004-\mathrm{N}^{\mathrm{O}} 2$ 
On peut directement chercher $u$ sous la forme

$$
u=u^{1}(r, \theta ; h) \exp \left(i \phi_{1}(r, \theta) / h\right)+u^{2}(r, \theta ; h) \exp \left(i \phi_{2}(r, \theta) / h\right)
$$

où $u^{j}(r, \theta ; h) \sim \sum_{k>0} u_{k}^{j}(r, \theta) h^{k}$ et montrer que, pour que $u$ soit solution non nulle du problème, les phases $\phi_{1}$ et $\phi_{2}$ doivent être solutions des équations de la proposition 3.2. C'est donc aussi une façon de retrouver l'existence de la variété caractéristique $\Sigma$ de l'opérateur de Neumann. De plus les amplitudes $u^{1}$ et $u^{2}$ doivent être non nulles toutes les deux pour que $u$ ne soit pas nulle; la solution $u$ vit donc sur les lagrangiennes $\Lambda_{1, r}$ et $\Lambda_{2, r}$. Cette méthode est élémentaire mais nécessite beaucoup de calculs et il semble très difficile ensuite de contrôler les fonctions $u_{k}^{j}(r, \theta)$ pour montrer que $u^{1}$ et $u^{2}$ sont des symboles analytiques.

Nous allons donc faire une construction BKW en deux temps en nous ramenant à un problème de Dirichlet à l'aide de l'opérateur de Dirichlet-Neumann.

Près de la variété caractéristique $\Sigma$, l'opérateur $N(h):=h \mathcal{N}\left(h^{-1}\right)$ est un opérateur pseudo-différentiel analytique. Nous montrons que les valeurs propres de son symbole principal ne se croisent pas au voisinage du complexifié de $\Sigma^{ \pm}$. Cela nous permet de diagonaliser $N(h)$ près du complexifié de $\Sigma^{ \pm}$et de construire une solution approchée $V^{ \pm}$de l'équation $N(h) V=0$, qui vit dans le complexifié de $\Sigma^{ \pm}$.

On construit ensuite une solution approchée du problème de Dirichlet

$$
\left(-h^{2} \Delta_{e}-1\right) u=0 \text { près de } \Gamma, \quad u=V^{ \pm} \text {sur } \Gamma .
$$

On diagonalise pour cela le système dans $\Omega$ et la condition au bord devient une perturbation $O(h)$ d'une condition de Dirichlet complètement découplée. Mais elle n'est pas découplée : c'est lié au fait que la solution $u$ que l'on construit vit à la fois sur les deux lagrangiennes associées aux deux vitesses d'onde. On adapte ensuite à notre cas la démonstration du théorème 9.3 dans [9].

4.2. Symboles analytiques. - Nous rappelons ici des notions introduites par Sjöstrand [9] qui nous seront utiles dans cette section.

DÉfinition. - Soit $\Omega$ un ouvert de $\mathbb{C}^{n}$. On définit l'espace $H_{0}^{\text {loc }}(\Omega)$ comme l'ensemble des fonctions $u(z ; h)$ holomorphes en $z$ dans $\Omega$ vérifiant la propriété suivante : pour tout compact $K \subset \Omega$ et tout $\varepsilon>0$, il existe une constante $C_{K, \varepsilon}>0$ telle que

$$
\forall z \in K, \quad|u(z ; h)| \leq C_{K, \varepsilon} \exp \left(\frac{\varepsilon}{h}\right) .
$$

Les éléments de $H_{0}^{\mathrm{loc}}(\Omega)$ sont appelés symboles analytiques. En particulier, $u$ sera dit symbole analytique d'ordre fini $m \in \mathbb{R}$, si pour tout compact $K \subset \Omega$, il existe une constante $C_{K}>0$ telle que

$$
\forall z \in K, \quad|u(z ; h)| \leq \frac{C_{K}}{h^{m}} .
$$

BULletin DE LA SOCIÉtÉ MATHÉMATIQUE DE FRANCE 
DÉfinition. - On ne distingue pas deux éléments de $H_{0}^{\text {loc }}(\Omega)$ dont la différence est exponentiellement petite. On dit que $u \sim v(u$ est équivalent à $v)$ si pour tout compact $K \subset \Omega$, il existe une constante $C_{K}>0$ et une constante $\varepsilon>0$ vérifiant

$$
\forall z \in K, \quad|u(z ; h)-v(z ; h)| \leq C_{K} \exp \left(-\frac{\varepsilon}{h}\right) .
$$

On note $S_{0}^{\text {loc }}(\Omega)$ l'espace $H_{0}^{\text {loc }}(\Omega)$ quotienté par cette relation équivalence.

DÉFINITION. - On appelle symbole analytique classique formel toute suite $\left(u_{k}(z)\right)_{k \in \mathbb{N}}$ de fonctions holomorphes sur $\Omega$ telle que pour tout compact $K \subset \Omega$ il existe une constante $C_{K}>0$ vérifiant

$$
\forall z \in K, \quad\left|u_{k}(z)\right| \leq C_{K}^{k+1} k^{k} .
$$

À tout symbole analytique classique formel $\left(u_{k}(z)\right)_{k \in \mathbb{N}}$, pour tout ouvert $\Omega^{\prime} \subset$ $K \subset \Omega$ où $K$ est un compact, on associe par la formule

$$
u(z ; h)=\sum_{0 \leq k \leq 1 /\left(e C_{K} h\right)} u_{k}(z) h^{k}
$$

un élément de $H_{0}^{\mathrm{loc}}\left(\Omega^{\prime}\right)$ et un unique élément de $S_{0}^{\mathrm{loc}}\left(\Omega^{\prime}\right)$ (indépendant de la constante $\left.C_{K}\right)$. Alors $u(z ; h)$ est un symbole d'ordre 0 .

DÉfinition. - On dit qu'un élément $u$ de $H_{0}^{\text {loc }}(\Omega)$ est un symbole analytique classique d'ordre $m$ s'il existe un suite $\left(u_{k}(z)\right)_{k \in \mathbb{N}}$ de fonctions holomorphes dans $\Omega$ telle que pour tout compact $K \subset \Omega$ il existe une constante $C_{K}$ vérifiant :

$$
\begin{aligned}
& \forall z \in K, \quad\left|u_{k}(z)\right| \leq C_{K}^{k+1} k^{k}, \\
& \forall N \in \mathbb{N}, \quad\left|u(z ; h)-h^{-m} \sum_{0 \leq k \leq N-1} u_{k}(z) h^{k}\right| \leq C_{K}^{N+1} N^{N} h^{N-m} .
\end{aligned}
$$

On dit que $u$ est elliptique si $u_{0}(z) \neq 0$ pour tout $z$ dans $\Omega$.

Semi-normes. - Soit $\Omega_{t}$, avec $0<t \leq t_{0}$, une famille de petits voisinages d'un point $\left(x_{0}, \xi_{0}\right)$ de $\mathbb{C}^{2 n}$ tels que $\Omega_{s} \subset \Omega_{t}$ pour $t<s$ et

$$
(y, \xi) \in \Omega_{s},|x-y| \leq s-t \Longrightarrow(x, \xi) \in \Omega_{t} .
$$

Alors pour $0<t<s \leq t_{0}, D_{x}^{\alpha}=1 / i^{|\alpha|} \partial_{x}^{\alpha}$ est un opérateur borné de l'espace $B\left(\Omega_{t}\right)$ des fonctions holomorphes bornées sur $\Omega_{t}$ dans l'espace $B\left(\Omega_{s}\right)$ de norme

$$
\left\|D_{x}^{\alpha}\right\|_{t, s} \leq \frac{C_{0}^{|\alpha|}|\alpha|^{|\alpha|}}{(s-t)^{|\alpha|}},
$$

où $C_{0}$ est une constante universelle ne dépendant que de $n$.

On associe à un symbole analytique classique $p(x, \xi ; h)$ d'ordre 0 un opérateur différentiel d'ordre infini

$$
\begin{aligned}
& \qquad A\left(x, \xi, D_{x}, ; h\right)=p\left(x, \xi+h D_{x} ; h\right)=\sum_{k \geq 0} h^{k} A_{k}\left(x, \xi, D_{x}\right) \\
& \text { et on a }\left\|A_{k}\right\|_{t, s} \leq C^{k+1} k^{k}(s-t)^{-k} . \\
& \text { томе } 132-2004-\mathrm{N}^{\mathrm{o}} 2
\end{aligned}
$$


Notons $f_{k}(A)$ la plus petite des constantes $\geq 0$ possibles vérifiant

$$
\left\|A_{k}\right\|_{t, s} \leq f_{k}(A) k^{k}(s-t)^{-k} .
$$

Pour $\mu>0$, on pose alors

$$
\|A\|_{\mu}=\sum_{k \geq 0} \mu^{k} f_{k}(A)
$$

cette quantité est finie pour un $\mu>0$.

Soit $u=\sum_{k>0} u_{k}$ un symbole où $\left(u_{k}(x, \xi)\right)_{k \in \mathbb{N}}$ une suite de fonctions holomorphes dans $\Omega_{0}$ vérifiant

$$
\left\|u_{k}\right\|_{\Omega_{t}}=\sup _{\Omega_{t}}\left|u_{k}\right| \leq C(k, u) k^{k} t^{-k}, \quad 0<t \leq t_{0} .
$$

Si l'on note $f_{k}(u)$ la plus petite constante $C(k, u) \geq 0$ dans l'inégalité ci-dessus, on pose pour $\mu>0$

$$
\|u\|_{\mu}=\sum_{k \geq 0} \mu^{k} f_{k}(u)
$$

\section{Propriétés}

- Le symbole $u$ est analytique dans $\Omega_{0}$ si et seulement si $\|u\|_{\mu}$ est fini pour un $\mu>0$.

- On a $\|A u\|_{\mu} \leq\|A\|_{\mu} \cdot\|u\|_{\mu}$.

DÉfinition. - Soient $p(x, \xi ; h)$ et $q(x, \xi ; h)$ des symboles analytiques classiques dans un ouvert $\Omega$ de $\mathbb{C}^{2 n}$. On définit le symbole composé par

$$
p \sharp q=\sum_{\alpha \in \mathbb{N}^{n}} \frac{1}{\alpha !}\left(\frac{h}{i}\right)^{|\alpha|} \partial_{\xi}^{\alpha} p \partial_{x}^{\alpha} q
$$

\section{Propriétés}

- Le composé de deux symboles analytiques classiques est un symbole analytique classique.

- Si $p$ est un symbole analytique classique elliptique, il existe un unique symbole analytique classique $q$, appelé symbole inverse de $p$, tel que $p \sharp q=q \sharp p=1$.

\subsection{Construction sur le bord}

4.3.1. Opérateur de Neumann près de $\Sigma$. — Soit $N(h):=h \mathcal{N}\left(h^{-1}\right)$. D'après le travail de Vodev [21] dans lequel est traité le cas analytique, près de $\Sigma, N(h)$ est un opérateur $h$-pseudodifférentiel analytique, qu'on notera $N_{e}(h)$.

Comme dans [16], nous calculons son symbole principal $n_{0}$ au voisinage de $\Sigma$, mais dans les coordonnées $(\theta, \eta)$, qui ont l'avantage d'être globales sur le 
revêtement de $T^{*} \Gamma$. Dans ces coordonnées, la zone elliptique $\mathcal{E}$ (qui contient $\Sigma$ ) est caractérisée par $\eta^{2} / R(\theta)^{2}>c_{1}^{-2}$. Posons

$$
\zeta_{1}=\sqrt{\frac{\eta^{2}}{R(\theta)^{2}}-c_{1}^{-2}} \text { et } \zeta_{2}=\sqrt{\frac{\eta^{2}}{R(\theta)^{2}}-c_{2}^{-2}} .
$$

On obtient $n_{0}=\mathcal{B} \mathcal{A}^{-1}$ où $\mathcal{B}=\left(\mathcal{B}_{i j}\right)_{\substack{1 \leq i \leq 2 \\ 1 \leq j \leq 2}}$ et $\mathcal{A}=\left(\mathcal{A}_{i j}\right)_{\substack{1 \leq i \leq 2 \\ 1 \leq j \leq 2}}$ sont définies par

$$
\begin{aligned}
\mathcal{A}_{11}= & c_{2}^{-2} \cos ^{2} \theta+c_{1}^{-2} \sin ^{2} \theta+i \sin (2 \theta)\left(\zeta_{1}-\zeta_{2}\right) \frac{\eta}{R(\theta)} \\
\mathcal{A}_{22}= & c_{1}^{-2} \cos ^{2} \theta+c_{2}^{-2} \sin ^{2} \theta+i \sin (2 \theta)\left(\zeta_{2}-\zeta_{1}\right) \frac{\eta}{R(\theta)} \\
\mathcal{A}_{12}= & \mathcal{A}_{21}=\frac{1}{2}\left(c_{2}^{-2}-c_{1}^{-2}\right) \sin (2 \theta)+i \cos (2 \theta)\left(\zeta_{2}-\zeta_{1}\right) \frac{\eta}{R(\theta)} \\
\mathcal{B}_{11}=\zeta_{2} \cos ^{2} \theta+\zeta_{1} \sin ^{2} \theta-i\left(1-c_{1}^{2} c_{2}^{-2}\right) \sin (2 \theta) \frac{\eta}{R(\theta)} & +2 c_{1}^{2} \cos (2 \theta)\left(\zeta_{1}-\zeta_{2}\right) \frac{\eta^{2}}{R(\theta)^{2}} \\
& +2 c_{1}^{2} \cos (2 \theta)\left(\zeta_{2}-\zeta_{1}\right) \frac{\eta^{2}}{R(\theta)^{2}} \\
\mathcal{B}_{22}= & \zeta_{1} \cos ^{2} \theta+\zeta_{2} \sin ^{2} \theta+i\left(1-c_{1}^{2} c_{2}^{-2}\right) \sin (2 \theta) \frac{\eta}{R(\theta)} \\
\mathcal{B}_{12}= & \frac{1}{2} \sin (2 \theta)\left(\zeta_{2}-\zeta_{1}\right)+i\left(\cos ^{2} \theta-\left(1-2 c_{1}^{2} c_{2}^{-2}\right) \sin ^{2} \theta\right) \frac{\eta}{R(\theta)} \\
& +2 c_{1}^{2} \sin (2 \theta)\left(\zeta_{1}-\zeta_{2}\right) \frac{\eta^{2}}{R(\theta)^{2}} v \\
\mathcal{B}_{21}= & \frac{1}{2} \sin (2 \theta)\left(\zeta_{2}-\zeta_{1}\right)+i\left(\left(1-2 c_{1}^{2} c_{2}^{-2}\right) \cos ^{2} \theta-\sin { }^{2} \theta\right) \frac{\eta}{R(\theta)} \\
& +2 c_{1}^{2} \sin (2 \theta)\left(\zeta_{1}-\zeta_{2}\right) \frac{\eta^{2}}{R(\theta)^{2}}
\end{aligned}
$$

On obtient

(3) $\operatorname{det}\left(n_{0}(\theta, \eta)\right)=-c_{1}^{4}\left(\frac{\eta^{2}}{R(\theta)^{2}}-\zeta_{1} \zeta_{2}\right)^{-1}\left[\left(c_{1}^{-2}-2 \frac{\eta^{2}}{R(\theta)^{2}}\right)^{2}-4 \zeta_{1} \zeta_{2} \frac{\eta^{2}}{R(\theta)^{2}}\right]$

$$
=-c_{1}^{4}\left(\frac{\eta^{2}}{R(\theta)^{2}}-\zeta_{1} \zeta_{2}\right)^{-1} \frac{\eta^{4}}{R(\theta)^{4}} \mathcal{R}\left(c_{1}^{-1} \frac{R(\theta)}{\eta}\right),
$$

où $\mathcal{R}$ est la fonction de Rayleigh. On a également

$$
\operatorname{Tr}\left(n_{0}(\theta, \eta)\right)=\left(c_{2}^{-2} \zeta_{1}+c_{1}^{-2} \zeta_{2}\right)\left(c_{1}^{-2} c_{2}^{-2}+\left(\zeta_{2}-\zeta_{1}\right)^{2} \frac{\eta^{2}}{R(\theta)^{2}}\right)^{-1} .
$$

On rappelle que $\mathcal{R}$ admet le nombre $c_{1}^{-1} c_{R}$ comme unique zéro dans ]0,1[ et que $\mathcal{R}^{\prime}\left(c_{1}^{-1} c_{R}\right) \neq 0$. De même $\mathcal{R}\left(-c_{1}^{-1} c_{R}\right)=0$ et $\mathcal{R}^{\prime}\left(-c_{1}^{-1} c_{R}\right) \neq 0$. On retrouve 
que le déterminant s'annule sur la variété

$$
\Sigma=\left\{(\theta, \eta) \in T^{*}(\Gamma) ; \eta^{2}=c_{R}^{-2} R(\theta)^{2}\right\} .
$$

$N_{e}(h)$ reste un opérateur $h$-pseudodifférentiel analytique près du complexifié $\Sigma=\left\{(\theta, \eta) \in T^{*}(\Gamma) ; \theta \in B_{a}\right.$ et $\left.\eta^{2}=c_{R}^{-2} R(\theta)^{2}\right\}$. Le déterminant de $n_{0}(\theta, \eta)$ s'annule sur le complexifié de $\Sigma$.

\subsubsection{Diagonalisation de l'opérateur de Neumann près du complexifié de $\Sigma$}

La matrice $2 \times 2 n_{0}(\theta, \eta)$ admet deux valeurs propres $a_{1}(\theta, \eta)$ et $a_{2}(\theta, \eta)$. D'après (3), (4) et le fait que $\pm c_{1}^{-1} c_{R}$ est un zéro simple de la fonction holomorphe $\mathcal{R}$, pour $(\theta, \eta)$ dans un voisinage du complexifié de $\Sigma$, on a

(5) $\quad a_{1}(\theta, \eta)=\widetilde{a}_{1}(\theta, \eta)\left(c_{R}^{2} \eta^{2}-R(\theta)^{2}\right) \quad$ où $\quad \widetilde{a}_{1}(\theta, \eta) \neq 0$ et $a_{2}(\theta, \eta) \neq 0$.

Posons $\psi(\theta)=\psi^{ \pm}(\theta)= \pm c_{R}^{-1} \int R(\theta) \mathrm{d} \theta$; donc $\psi$ est telle que

$$
\operatorname{det}\left(n_{0}\left(\theta, \partial_{\theta} \psi(\theta)\right)\right)=0 \text {. }
$$

Posons alors

$$
N_{e}^{\psi}(h)=\exp (-i \psi / h) N_{e}(h) \exp (i \psi / h) .
$$

$N_{e}^{\psi}(h)$ est aussi un opérateur $h$-pseudodifférentiel analytique. Notons $n^{\psi}$ son symbole et $n_{0}^{\psi}$ son symbole principal.

La matrice $n_{0}^{\psi}(\theta, \eta)=n_{0}\left(\theta, \eta+\partial_{\theta} \psi(\theta)\right)=n_{0}\left(\theta, \eta \pm c_{R}^{-1} R(\theta)\right)$ est une matrice $2 \times 2$ ayant deux valeurs propres $a_{1}^{\psi}$ et $a_{2}^{\psi}$. Nous allons montrer que ces valeurs propres ne se croisent pas dans un certain domaine.

Lemme 4.1. - Il existe un voisinage complexe $W$ de 0 et une constante $C_{0}>0$ tels que

$$
\forall(\theta, \eta) \in B_{a} \times W, \quad\left|a_{1}^{\psi}(\theta, \eta)-a_{2}^{\psi}(\theta, \eta)\right| \geq C_{0} .
$$

Démonstration. - $a_{1}^{\psi}(\theta, \eta)=\widetilde{a}_{1}\left(\theta, \eta \pm c_{R}^{-1} R(\theta)\right)\left(c_{R}^{2} \eta^{2} \pm 2 \eta c_{R} R(\theta)\right)$ est aussi proche de 0 que l'on veut pourvu que $W$ soit un voisinage de 0 assez petit. D'après (4), $a_{1}^{\psi}+a_{2}^{\psi}$ ne s'annule pas dans $B_{a} \times W$ pourvu que $W$ soit assez petit; d'où le résultat.

Il existe donc une matrice $T(\theta, \eta)$ analytique dans $B_{a} \times W$ où $W$ est un voisinage complexe de 0 , telle que

$$
T^{-1}(\theta, \eta) n_{0}^{\psi}(\theta, \eta) T(\theta, \eta)=\left(\begin{array}{cc}
a_{1}^{\psi}(\theta, \eta) & 0 \\
0 & a_{2}^{\psi}(\theta, \eta)
\end{array}\right) .
$$

L'opérateur $T^{-1}\left(\theta, h D_{\theta}\right) N_{e}^{\psi}(h) T\left(\theta, h D_{\theta}\right)$ de symbole

$$
R(\theta, \eta ; h)=T^{-1}(\theta, \eta) \sharp n^{\psi}(\theta, \eta ; h) \sharp T(\theta, \eta)
$$

a pour symbole principal

$$
\left(\begin{array}{cc}
a_{1}^{\psi}(\theta, \eta) & 0 \\
0 & a_{2}^{\psi}(\theta, \eta)
\end{array}\right)
$$

BULLETIN DE LA SOCIÉtÉ MATHÉMATIQUE DE FRANCE 
Nous allons maintenant nous ramener à la dimension 1 pour pouvoir appliquer les résultats de Sjöstrand [9]. Nous allons rappeler une proposition de L. Nédélec [8] dont la démonstration suit Helffer-Sjöstrand [5] (section 3.2) :

Proposition 4.2 (voir [8]). - Soit $R(x, \xi ; h)$ un symbole analytique classique formel d'ordre 0 sur un ouvert $\Omega \times V$ de $\mathbb{C}^{2}$, de symbole principal

$$
r(x, \xi)=\left(\begin{array}{cc}
r_{1,1}(x, \xi) & 0 \\
0 & r_{2,2}(x, \xi)
\end{array}\right)
$$

et vérifiant de plus

$$
\exists C_{0}>0, \forall(x, \xi) \in \Omega \times V, \quad\left|r_{1,1}(x, \xi)-r_{2,2}(x, \xi)\right| \geq C_{0} .
$$

Alors il existe un symbole analytique classique elliptique d'ordre $0 U(x, \xi ; h)$ sur $\Omega \times V$ tel que

$$
U^{-1}(x, \xi ; h) \sharp R(x, \xi ; h) \sharp U(x, \xi ; h)=\left(\begin{array}{cc}
R_{1}(x, \xi ; h) & 0 \\
0 & R_{2}(x, \xi ; h)
\end{array}\right)
$$

où $R_{j}(x, \xi ; h)$ a pour symbole principal $r_{j, j}(x, \xi ; h)$ pour $j=1,2$.

D'après la proposition 4.2 et le lemme 4.1 il existe un symbole analytique classique d'ordre $0 U(\theta, \eta ; h)$ dans $B_{a} \times W$ tel que

$$
\begin{aligned}
U^{-1}(\theta, \eta ; h) & \sharp R(\theta, \eta ; h) \sharp U(\theta, \eta ; h) \\
= & U^{-1}(\theta, \eta ; h) \sharp T^{-1}(\theta, \eta) \sharp n^{\psi}(\theta, \eta ; h) \sharp T(\theta, \eta) \sharp U(\theta, \eta ; h) \\
& =\left(\begin{array}{cc}
R_{1}(\theta, \eta ; h) & 0 \\
0 & R_{2}(\theta, \eta ; h)
\end{array}\right)
\end{aligned}
$$

où $R_{1}(\theta, \eta ; h)$ et $R_{2}(\theta, \eta ; h)$ sont des symboles analytiques classiques ayant respectivement pour symboles principaux $a_{1}^{\psi}(\theta, \eta)$ et $a_{2}^{\psi}(\theta, \eta)$. On a donc

(6) $U^{-1}\left(\theta, h D_{\theta} ; h\right) T^{-1}\left(\theta, h D_{\theta}\right) N_{e}^{\psi}(h) T\left(\theta, h D_{\theta}\right) U\left(\theta, h D_{\theta} ; h\right)$

$$
=\left(\begin{array}{cc}
R_{1}\left(\theta, h D_{\theta} ; h\right) & 0 \\
0 & R_{2}\left(\theta, h D_{\theta} ; h\right)
\end{array}\right) .
$$

D'après (5), pour $\theta$ dans $B_{a}$, on a $a_{2}^{\psi}(\theta, 0)=a_{2}\left(\theta, \pm c_{R}^{-1} R(\theta)\right) \neq 0$ et

$$
\left\{\begin{array}{l}
a_{1}^{\psi}(\theta, 0)=a_{1}\left(\theta, \pm c_{R}^{-1} R(\theta)\right)=0, \\
\partial_{\eta} a_{1}^{\psi}(\theta, 0)= \pm 2 c_{R} R(\theta) \tilde{a}_{1}\left(\theta, \pm c_{R}^{-1} R(\theta)\right) \neq 0 .
\end{array}\right.
$$

D'après les résultats de Sjöstrand [9] sur les symboles analytiques en dimension 1 , il existe un symbole analytique classique non nul d'ordre $0 r_{1}(\theta ; h)$ dans $B_{a}$ tel que

$$
R_{1}\left(\theta, h D_{\theta} ; h\right) r_{1}(\theta ; h)=0 \text {. }
$$

TOME $132-2004-\mathrm{N}^{\mathrm{O}} 2$ 
Si l'on note $r_{1}=\sum_{k=0}^{+\infty} r_{1, k} h^{k}$, alors $r_{1,0}$ ne s'annule pas dans la bande $B_{a}$ d'après (7). Si l'on pose

$$
v(\theta ; h)=T\left(\theta, h D_{\theta}\right) U\left(\theta, h D_{\theta} ; h\right)\left(\begin{array}{c}
r_{1}(\theta ; h) \\
0
\end{array}\right)
$$

alors $v(\theta ; h)$ est un symbole analytique classique non nul puisque $T(\theta, \eta)$ et $U(\theta, \eta ; h)$ sont elliptiques, et avec (6) on a

$$
N_{e}^{\psi}(h) v(\theta ; h)=0 .
$$

On obtient la proposition suivante :

Proposition 4.3. - Il existe un symbole analytique classique d'ordre $0 v^{ \pm}(\theta ; h)$ dans $B_{a}$ tel que

$$
N_{e}^{\psi^{ \pm}}(h) v^{ \pm}(\theta ; h)=0 .
$$

Remarque. - on a $v^{-}=\bar{v}^{+}$puisque $\psi^{-}=-\psi^{+}$.

Proposition 4.4. - Il existe un symbole $c^{ \pm}(h)=\sum_{k \geq 0} c_{k} h^{k}$ analytique classique tel que

$$
v^{ \pm}(\theta+2 \pi ; h)=c^{ \pm}(h) v^{ \pm}(\theta ; h) .
$$

Démonstration. - Nous allons montrer dans un premier temps qu'il existe un symbole analytique classique $c(h)$ vérifiant

$$
r_{1}(\theta+2 \pi ; h)=c(h) r_{1}(\theta ; h) \text {. }
$$

Pour cela, nous allons préciser les équations de transport apparaissant dans la résolution de l'équation $R_{1}\left(\theta, h D_{\theta} ; h\right) r_{1}(\theta ; h)=0$ (voir [9]). Notons

$$
r_{1}(\theta ; h)=\sum_{k \geq 0} r_{1, k}(\theta) h^{k} \quad \text { et } \quad R_{1}(\theta, \eta ; h)=\sum_{k \geq 0} R_{1, k}(\theta, \eta) h^{k} .
$$

Précisons que $R_{1,0}=a_{1}^{\psi}$. On a

$$
\begin{aligned}
R_{1}\left(\theta, h D_{\theta} ; h\right) & =\sum_{k+\alpha \geq 0} h^{k+\alpha} \partial_{\eta}^{\alpha} R_{1, k}(\theta, 0) D_{\theta}^{\alpha} \\
& =\partial_{\eta} R_{1,0}(\theta, 0) h D_{\theta}+h R_{1,1}(\theta, 0)+\sum_{j \geq 2} h^{j} A_{j}
\end{aligned}
$$

où $A_{j}$ est un opérateur différentiel d'ordre fini ne dépendant pas de $h$. On obtient donc comme équations de transport

$$
\partial_{\eta} R_{1,0}(\theta, 0) D_{\theta} r_{1,0}(\theta)+R_{1,1}(\theta, 0) r_{1,0}(\theta)=0
$$

et pour $k \geq 1$

$$
\partial_{\eta} R_{1,0}(\theta, 0) D_{\theta} r_{1, k}(\theta)+R_{1,1}(\theta, 0) r_{1, k}(\theta)=-\sum_{2 \leq j \leq k+1} A_{j} r_{1, k+1-j}(\theta) .
$$

BULlETIN DE LA SOCIÉTÉ MATHÉMATIQUE DE FRANCE 
$r_{1,0}(\theta+2 \pi)$ et $r_{1,0}(\theta)$ sont tous les deux solutions de l'équation homogène (9), donc il existe un nombre $c_{0}$ tel que

$$
r_{1,0}(\theta+2 \pi)=c_{0} r_{1,0}(\theta) .
$$

Supposons maintenant qu'il existe des nombres $c_{0}, \ldots, c_{m}$ tels que

$$
r_{1, j}(\theta+2 \pi)=\sum_{0 \leq k \leq j} c_{k} r_{1, j-k}(\theta) \quad \text { pour } j=0, \ldots, m
$$

Puisque les coefficients des équations (9) et (10) sont $2 \pi$-périodiques en la variable $\theta$, on a

$$
\begin{aligned}
\partial_{\eta} R_{1,0}(\theta, 0) & D_{\theta} r_{1, m+1}(\theta+2 \pi)+R_{1,1}(\theta, 0) r_{1, m+1}(\theta+2 \pi) \\
& =-\sum_{2 \leq \ell \leq m+2} A_{\ell} r_{1, m+2-\ell}(\theta+2 \pi) \\
& =-\sum_{2 \leq \ell \leq m+2} A_{\ell} \sum_{0 \leq k \leq m+2-\ell} c_{k} r_{1, m+2-\ell-k}(\theta) \\
& =-\sum_{0 \leq k \leq m} c_{k} \sum_{2 \leq \ell \leq m+2-k} A_{\ell} r_{1, m+2-\ell-k}(\theta) .
\end{aligned}
$$

Donc $r_{1, m+1}(\theta+2 \pi)-\sum_{0 \leq k \leq m} c_{k} r_{1, m+1-k}(\theta)$ est solution de l'équation homogène $(9)$; on en déduit qu'il existe un nombre $c_{m+1}$ tel que

$$
r_{1, m+1}(\theta+2 \pi)-\sum_{0 \leq k \leq m} c_{k} r_{1, m+1-k}(\theta)=c_{m+1} r_{1,0}(\theta)
$$

c'est-à-dire

$$
r_{1, m+1}(\theta+2 \pi)=\sum_{0 \leq k \leq m+1} c_{k} r_{1, m+1-k}(\theta) .
$$

La formule (11) est donc vraie pour tout $m$ dans $\mathbb{N}$. Si on pose $c(h)=\sum_{k \geq 0} c_{k} h^{k}$, alors on a formellement

$$
r_{1}(\theta+2 \pi ; h)=c(h) r_{1}(\theta ; h) .
$$

De plus $r_{1,0}(\theta)$, solution de l'équation homogène (9), ne s'annule pas. Donc $r_{1}(\theta ; h)$ et $r_{1}(\theta+2 \pi ; h)$ sont des symboles analytiques classiques elliptiques. Il s'ensuit que

$$
c(h)=r_{1}(\theta+2 \pi ; h) \sharp r_{1}(\theta ; h)^{-\sharp 1}
$$

où $r_{1}(\theta ; h)^{-\sharp 1}$ désigne le symbole analytique inverse de $r_{1}(\theta ; h)$. Le symbole $c(h)$ est donc un symbole analytique classique. Et d'après (8),

$$
v(\theta+2 \pi ; h)=c(h) v(\theta ; h) .
$$

Remarque. - On a évidemment $c^{-}(h)=\overline{c^{+}(h)}$. 
4.4. Construction près du bord. - Soient $\phi_{1}^{ \pm}$et $\phi_{2}^{ \pm}$les phases construites dans la proposition 3.2 et vérifiant

$$
\phi_{1}^{ \pm}(0, \theta)=\phi_{2}^{ \pm}(0, \theta)=\psi^{ \pm}(\theta)
$$

et $\Lambda_{j, r}^{ \pm}$les lagrangiennes relevées construites dans la section 3. La notation $Q(h)$ désigne l'opérateur $-h^{2} \Delta_{e}-1$ dans les coordonnées $(r, \theta)$.

Proposition 4.5. - Soit $v^{ \pm}=\left(\begin{array}{l}v_{1} \\ v_{2}\end{array}\right)$ le symbole construit dans la proposition 4.3. Il existe des symboles analytiques classiques $u^{1, \pm}$ et $u^{2, \pm}$ tels que

$$
\left\{\begin{array}{lll}
\exp \left(-i \phi_{1}^{ \pm} / h\right) Q(h) \exp \left(i \phi_{1}^{ \pm} / h\right) u^{1}=0 & \text { dans } \Pi\left(\Lambda_{1, r}^{ \pm}\right), \\
\exp \left(-i \phi_{2}^{ \pm} / h\right) Q(h) \exp \left(i \phi_{2}^{ \pm} / h\right) u^{2}=0 & \text { dans } \Pi\left(\Lambda_{2, r}^{ \pm}\right), \\
u^{1}+u^{2}=v, & \text { sur } \Gamma,
\end{array}\right.
$$

où $\Gamma$ désigne ici le complexifié du revêtement universel de $\Gamma$.

Démonstration. - Puisque l'on travaille localement, on peut tout exprimer dans les coordonnées euclidiennes. Réduisons d'abord le symbole principal $q(\xi)$ de $Q(h)$. Celui-ci a deux valeurs propres $q_{1}(\xi)=c_{1}^{2} \xi^{2}-1$ et $q_{2}(\xi)=c_{2}^{2} \xi^{2}-1$. Soit $V(\xi)=\left(\begin{array}{cc}\xi_{2} & \xi_{1} \\ -\xi_{1} & \xi_{2}\end{array}\right)$. On a

$$
V(\xi)^{-1}=\frac{1}{\xi^{2}}\left(\begin{array}{cc}
\xi_{2} & -\xi_{1} \\
\xi_{1} & \xi_{2}
\end{array}\right) \quad \text { et } \quad V(\xi)^{-1} q(\xi) V(\xi)=\left(\begin{array}{cc}
q_{1}(\xi) & 0 \\
0 & q_{2}(\xi)
\end{array}\right)
$$

c'est-à-dire

$$
V(\xi)^{-1} \sharp q(\xi) \sharp V(\xi)=\left(\begin{array}{cc}
q_{1}(\xi) & 0 \\
0 & q_{2}(\xi)
\end{array}\right) .
$$

On a donc

$$
\begin{gathered}
\underbrace{\exp \left(-i \phi_{j} / h\right) V\left(h D_{x}\right)^{-1} \exp \left(i \phi_{j} / h\right)}_{=V_{j}^{-1}} \underbrace{\exp \left(-i \phi_{j} / h\right) Q(h) \exp \left(i \phi_{j} / h\right)}_{:=Q_{j}} \\
\times \underbrace{\exp \left(-i \phi_{j} / h\right) V\left(h D_{x}\right) \exp \left(i \phi_{j} / h\right)}_{:=V_{j}} \\
=\exp \left(-i \phi_{j} / h\right)\left(\begin{array}{cc}
q_{1}\left(h D_{x}\right) & 0 \\
0 & q_{2}\left(h D_{x}\right)
\end{array}\right) \exp \left(i \phi_{j} / h\right)=\left(\begin{array}{cc}
q_{1 j}\left(x, h D_{x}\right) & 0 \\
0 & q_{2 j}\left(x, h D_{x}\right)
\end{array}\right)
\end{gathered}
$$

où

$$
q_{k j}(x, \xi)=c_{k}^{2}\left(\xi+\nabla \phi_{j}\right)^{2}=2 c_{k}^{2} \nabla \phi_{j} \cdot \xi+c_{k}^{2} \xi^{2}+c_{k}^{2} c_{j}^{-2}-1
$$

On cherche $u^{j}$ symbole analytique d'ordre 0 tel que $Q_{j}\left(x, h D_{x}\right) u^{j}=0$. Puisque $\operatorname{det} V_{j}(x, 0)=\operatorname{det} V\left(\nabla \phi_{j}\right)=\left|\nabla \phi_{j}\right|^{2} \neq 0$, on peut poser $u^{j}=V_{j}\left(\begin{array}{l}\omega_{j 1} \\ \omega_{j 2}\end{array}\right)$, et on se ramène à chercher des symboles analytiques d'ordre $0 \omega_{j k}$ vérifiant

$$
q_{k j}\left(x, h D_{x}\right) \omega_{j k}=0 .
$$

On a $q_{k j}(x, 0) \neq 0$ si $k \neq j$; donc si $k \neq j$, on a $\omega_{j k}=0$.

BULLETIN DE LA SOCIÉtÉ MATHÉMATIQUE DE FRANCE 
La condition au bord s'écrit alors

$$
V_{1}\left(\begin{array}{c}
\omega_{11} \\
0
\end{array}\right)+\left.V_{2}\left(\begin{array}{c}
0 \\
\omega_{22}
\end{array}\right)\right|_{r=0}=\left(\begin{array}{c}
v_{1} \\
v_{2}
\end{array}\right)
$$

Or on a

$$
V_{1}\left(\begin{array}{c}
\omega_{11} \\
0
\end{array}\right)+V_{2}\left(\begin{array}{c}
0 \\
\omega_{22}
\end{array}\right)=[\underbrace{\left(\begin{array}{cc}
\partial_{x_{2}} \phi_{1} & \partial_{x_{1}} \phi_{2} \\
-\partial_{x_{1}} \phi_{1} & \partial_{x_{2}} \phi_{2}
\end{array}\right)}_{:=A}+h \underbrace{\left(\begin{array}{cc}
D_{x_{2}} & D_{x_{1}} \\
-D_{x_{1}} & D_{x_{2}}
\end{array}\right)}_{:=C}]\left(\begin{array}{c}
\omega_{11} \\
\omega_{22}
\end{array}\right) .
$$

Pour $r=0$, on a

$$
\begin{aligned}
\operatorname{det} A & =\partial_{x_{2}} \phi_{1} \partial_{x_{2}} \phi_{2}+\partial_{x_{1}} \phi_{1} \partial_{x_{1}} \phi_{2}=\partial_{r} \phi_{1} \partial_{r} \phi_{2}+\frac{1}{R(\theta)^{2}} \partial_{\theta} \phi_{1} \partial_{\theta} \phi_{2} \\
& =-\left(c_{R}^{-2}-c_{1}^{-2}\right)^{\frac{1}{2}}\left(c_{R}^{-2}-c_{2}^{-2}\right)^{\frac{1}{2}}+c_{R}^{-2} \neq 0
\end{aligned}
$$

$A_{\mid r=0}$ est donc inversible et analytique, et (12) s'écrit alors

$$
\left\{\begin{array}{l}
q_{11}\left(x, h D_{x}\right) \omega_{11}=0, \quad q_{22}\left(x, h D_{x}\right) \omega_{22}=0, \\
\left(\begin{array}{l}
\omega_{11} \\
\omega_{22}
\end{array}\right)+\left.h E\left(\begin{array}{l}
\omega_{11} \\
\omega_{22}
\end{array}\right)\right|_{r=0}=v^{\prime}
\end{array}\right.
$$

où $E=\left(A_{\mid r=0}\right)^{-1} C$ est un opérateur différentiel matriciel d'ordre 1 indépendant de $h$, et $v^{\prime}=\left(A_{\mid r=0}\right)^{-1} v$ est un symbole analytique d'ordre 0 puisque $v$ en est un.

On déduit de $q_{k k}(x, \xi)=2 c_{k}^{2} \nabla \phi_{j} \cdot \xi+c_{k}^{2} \xi^{2}$ que l'on a $q_{k k}(x, 0)=0$, $\partial_{\xi} q_{k k}(x, 0) \neq 0$ et que $\partial_{\xi} q_{k k}(x, 0) \cdot \partial_{x}$ est transverse à $\Gamma$. À une perturbation près, le système (13) serait entièrement découplé et le théorème 9.3 de [9] s'appliquerait. Il donnerait l'existence de symboles analytiques classiques d'ordre 0 , $\omega_{11}$ et $\omega_{22}$, vérifiant (13). On va adapter la démonstration de ce théorème à notre cas de figure.

Si l'on regroupe les termes avec la même homogénéité dans (13), on obtient une suite d'équations de transport qui déterminent $\omega_{11}$ et $\omega_{22}$ de manière unique. On va montrer que l'on obtient des symboles analytiques classiques.

On se place près d'un point $x_{0}$ du bord. Soit $\left(r_{j}, \beta_{j}\right)$ les coordonnées relevant les champs de vecteur $\nabla \phi_{j} \cdot \partial_{x}$ et tels que $\Gamma$ soit donnée par $r_{j}=0$ et $x_{0}$ par $r_{j}=\beta_{j}=0$. Pour $0 \leq t \leq t_{0}$, on définit

$$
\Omega_{t}^{j}=\left\{x \in \mathbb{C}^{2} ; \frac{\left|\beta_{j}\right|}{T_{0}-T_{0} t / t_{0}}+\frac{\left|r_{j}\right|}{t_{0}-t} \leq 1\right\}
$$

où $T_{0}$ et $t_{0}>0$ sont assez petits pour que $\Omega_{0} \cap \Gamma$ soit contenu dans le domaine de $v^{\prime}$. Soit $a$ holomorphe sur $\Omega_{0}$ et vérifiant pour $k>1$

$$
\sup _{\Omega_{t}^{j}}|a| \leq C_{j}(a, k) t^{-k}, \quad 0<t \leq t_{0} .
$$

TOME $132-2004-\mathrm{N}^{\mathrm{O}} 2$ 
Si l'on pose

on rappelle que

$$
\left(\partial_{r_{j}}\right)^{-1} a=\int_{0}^{r_{j}} a\left(s, \beta_{j}\right) \mathrm{d} s
$$

$$
\sup _{\Omega_{t}^{j}}\left|\left(\partial_{r_{j}}\right)^{-1} a\right| \leq \frac{C_{j}(a, k)}{(k-1) t^{k-1}} .
$$

On en déduit que si $a=\sum_{k \geq 2} a_{k} h^{k}$ est un symbole d'ordre -2 et que l'on pose $b=\left(h \partial_{r_{j}}\right)^{-1} a$, alors

$$
\|b\|_{\mu}^{j} \leq \frac{2 e}{\mu}\|a\|_{\mu}^{j} .
$$

Dans les nouvelles coordonnées, (13) devient

$$
\begin{aligned}
& \partial_{r_{1}} \omega_{11}=h^{-1} A^{1} \omega_{11}, \quad \partial_{r_{2}} \omega_{22}=h^{-1} A^{2} \omega_{22}, \\
& \omega_{11 \mid \Gamma}=-h E_{11} \omega_{11}-h E_{12} \omega_{22 \mid \Gamma}+v_{1}^{\prime}, \\
& \omega_{22 \mid \Gamma}=-h E_{21} \omega_{11}-h E_{22} \omega_{22 \mid \Gamma}+v_{2}^{\prime},
\end{aligned}
$$

où $E_{i j},(i, j) \in\{1,2\}$ sont des opérateurs différentiels d'ordre 1 à coefficients analytiques indépendants de $h$, et $h^{-2} A^{j}$, avec $j \in\{1,2\}$, sont des opérateurs différentiels d'ordre 2 à coefficients analytiques indépendants de $h$. On obtient donc

$$
\begin{aligned}
& \omega_{11}=\left(h \partial_{r_{1}}\right)^{-1}\left(A^{1} \omega_{11}\right)-h E_{11} \omega_{\left.11\right|_{r_{1}=0}}-h E_{12} \omega_{22 \mid r_{1}=0}+v_{1}^{\prime}, \\
& \omega_{22}=\left(h \partial_{r_{2}}\right)^{-1}\left(A^{2} \omega_{22}\right)-\left.h E_{21} \omega_{11}\right|_{r_{2}=0}-\left.h E_{22} \omega_{22}\right|_{r_{2}=0}+v_{2}^{\prime} .
\end{aligned}
$$

Puisque $\left\|A^{j}\right\|_{\mu}^{j}=O\left(\mu^{2}\right)$, on a

(14) $\left\|\left(h \partial_{r_{j}}\right)^{-1}\left(A^{j} \omega_{j j}\right)\right\|_{\mu}^{j} \leq \frac{2 e}{\mu}\left\|A^{j} \omega_{j j}\right\|_{\mu}^{j} \leq \frac{2 e}{\mu}\left\|A^{j}\right\|_{\mu}^{j} \cdot\left\|\omega_{j j}\right\|_{\mu}^{j} \leq O(\mu)\left\|\omega_{j j}\right\|_{\mu}^{j}$.

On a aussi

$$
\left\|h E_{j j} \omega_{j j \mid r_{j}=0}\right\|_{\mu}^{j} \leq\left\|h E_{j j} \omega_{j j}\right\|_{\mu}^{j}=O(\mu)\left\|\omega_{j j}\right\|_{\mu}^{j} .
$$

On peut s'arranger pour que sur $\Gamma$ on ait $\beta_{1}=\beta_{2}$, donc $\Omega_{t}^{1} \cap \Gamma=\Omega_{t}^{2} \cap \Gamma$, ce qui permet d'affirmer que

$$
\|\cdot \mid \Gamma\|_{\mu}^{j} \leq\|\cdot\|_{\mu}^{k} \quad \text { pour } \quad(j, k) \in\{1,2\} .
$$

On en déduit

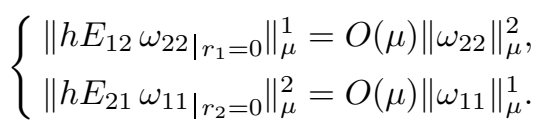

On déduit de (14), (15) et (16) que

$$
\begin{aligned}
& \left\|\omega_{11}\right\|_{\mu}^{1} \leq O(\mu)\left(\left\|\omega_{11}\right\|_{\mu}^{1}+\left\|\omega_{22}\right\|_{\mu}^{2}\right)+\left\|v_{1}^{\prime}\right\|_{\mu}^{1}, \\
& \left\|\omega_{22}\right\|_{\mu}^{2} \leq O(\mu)\left(\left\|\omega_{11}\right\|_{\mu}^{1}+\left\|\omega_{22}\right\|_{\mu}^{2}\right)+\left\|v_{2}^{\prime}\right\|_{\mu}^{2},
\end{aligned}
$$

BULLETIN DE LA SOCiÉTÉ MATHÉMATIQUe DE FRANCE 
d'où

$$
\left\|\omega_{11}\right\|_{\mu}^{1}+\left\|\omega_{22}\right\|_{\mu}^{2} \leq O(\mu)\left(\left\|\omega_{11}\right\|_{\mu}^{1}+\left\|\omega_{22}\right\|_{\mu}^{2}\right)+\left\|v_{1}^{\prime}\right\|_{\mu}^{1}+\left\|v_{2}^{\prime}\right\|_{\mu}^{2}
$$

Les symboles $v_{1}^{\prime}$ et $v_{2}^{\prime}$ étant analytiques, on a $\left\|v_{1}^{\prime}\right\|_{\mu}^{1}+\left\|v_{2}^{\prime}\right\|_{\mu}^{2}<+\infty$ et finalement on obtient que $\left\|\omega_{11}\right\|_{\mu}^{1}+\left\|\omega_{22}\right\|_{\mu}^{2}<+\infty$ pour $\mu$ assez petit, d'où $\omega_{11}$ et $\omega_{22}$ sont des symboles analytiques dans un voisinage du bord $\Gamma$. Or on a

$$
q_{11}\left(x, h D_{x}\right) \omega_{11}=0, \quad q_{22}\left(x, h D_{x}\right) \omega_{22}=0
$$

où $q_{j j}\left(x, h D_{x}\right)=2 c_{j}^{2} \nabla \phi_{j} \cdot h D_{x}+c_{j}^{2} h^{2} D_{x}^{2}$ pour $j=1,2$. En résolvant les équations de transport le long des courbes de $\nabla \phi_{j}$, on obtient des symboles $\omega_{j j}$ dans $\Pi\left(\Lambda_{j, r}^{ \pm}\right)$et ils sont analytiques d'après le théorème 9.3 de [9]. Et puisque $u^{1}=V_{1}\left(\begin{array}{c}\omega_{11} \\ 0\end{array}\right)$ et $u^{2}=V_{2}\left(\begin{array}{c}0 \\ \omega_{22}\end{array}\right), u^{j}$ est un symbole analytique dans $\Pi\left(\Lambda_{j, r}^{ \pm}\right)$ pour $j=1,2$.

Proposition 4.6. - Pour $j=1,2$, on a $u^{j}(r, \theta+2 \pi ; h)=c(h) u^{j}(r, \theta ; h)$ où $c(h)$ est le symbole analytique défini dans la proposition 4.4 .

Démonstration. - Commençons par préciser les équations de transport obtenues en regroupant les termes avec la même homogénéité dans (13). Si $\omega_{j j}=\sum_{k \geq 0} \omega_{k}^{j} h^{k}, v^{\prime}=\sum_{k \geq 0} v_{k}^{\prime} h^{k}$ et $\omega_{k}=\left(\begin{array}{c}\omega_{k}^{1} \\ \omega_{k}^{2}\end{array}\right)$, on a

$$
X_{j} \omega_{0}^{j}=0(j=1,2),\left.\quad \omega_{0}\right|_{r=0}=v_{0}^{\prime}
$$

et pour $k \geq 1$

$$
\left\{\begin{array}{l}
X_{j} \omega_{k}^{j}=\Delta_{r, \theta} \omega_{k-1}^{j} \quad(j=1,2), \\
\omega_{\left.k\right|_{r=0}}=-E \omega_{k-\left.1\right|_{r=0}}+v_{k}^{\prime}
\end{array}\right.
$$

où $\Delta_{r, \theta}$ est le laplacien dans les coordonnées $(r, \theta)$ et

$$
X_{j}=2 \partial_{r} \phi_{j}(r, \theta) \partial_{r}+\frac{2}{[r+R(\theta)]^{2}} \partial_{\theta} \phi_{j}(r, \theta) \partial_{\theta}
$$

Comme $\partial_{\theta} \phi_{j}$ est $2 \pi$-périodique en la variable $\theta,\left(A_{\mid r=0}\right)^{-1}$ l'est aussi; on en déduit, avec la proposition 4.4, que $v^{\prime}(\theta+2 \pi ; h)=c(h) v^{\prime}(\theta ; h)$. On a

$$
\begin{gathered}
X_{j} \omega_{0}^{j}(r, \theta+2 \pi)=0 \quad(j=1,2), \\
\omega_{0}(0, \theta+2 \pi)=v_{0}^{\prime}(\theta+2 \pi)=c_{0} v_{0}^{\prime}(\theta) .
\end{gathered}
$$

TOME $132-2004-\mathrm{N}^{\mathrm{O}} 2$ 
L'unicité de la solution dans (17) implique $\omega_{0}(r, \theta+2 \pi)=c_{0} \omega_{0}(r, \theta)$. Supposons maintenant que $\omega_{m}(r, \theta+2 \pi)=\sum_{0 \leq k \leq m} c_{k} \omega_{m-k}(r, \theta)$; on a alors

$$
\begin{aligned}
X_{j} \omega_{m+1}^{j}(r, \theta+2 \pi) & =\Delta_{r, \theta} \omega_{m}^{j}(r, \theta+2 \pi) \\
& =\sum_{0 \leq k \leq m} c_{k} \Delta_{r, \theta} \omega_{m-k}^{j}(r, \theta) \quad(j=1,2), \\
\omega_{m+1}(0, \theta+2 \pi) & =-E \omega_{m}(0, \theta+2 \pi)+v_{m+1}^{\prime}(\theta+2 \pi) \\
& =-\sum_{0 \leq k \leq m} c_{k} E \omega_{m-k}(0, \theta)+\sum_{0 \leq k \leq m+1} c_{k} v_{m+1-k}^{\prime}(\theta) \\
& =\sum_{0 \leq k \leq m} c_{k}\left(-E \omega_{m-k}(0, \theta)+v_{m+1-k}^{\prime}(\theta)\right)+c_{m+1} v_{0}^{\prime}(\theta) .
\end{aligned}
$$

D'après l'unicité des solutions dans (17) et (18) on obtient

$$
\begin{aligned}
\omega_{m+1}(r, \theta+2 \pi) & =c_{m+1} \omega_{0}(r, \theta)+\sum_{0 \leq k \leq m} c_{k} \omega_{m+1-k}(r, \theta) \\
& =\sum_{0 \leq k \leq m+1} c_{k} \omega_{m+1-k}(r, \theta) .
\end{aligned}
$$

On a donc $\omega_{j j}(r, \theta+2 \pi ; h)=c(h) \omega_{j j}(r, \theta ; h)$ et $V_{j}$ étant des opérateurs différentiels à coefficients $2 \pi$-périodiques en la variable $\theta$, on peut conclure.

On énonce une proposition qui signifie que la solution BKW $u$ vit bien à la fois sur les deux lagrangiennes :

Proposition 4.7. - $u_{0}^{1}$ ne s'annule pas dans $\Pi\left(\Lambda_{1, r}^{ \pm}\right)$et $u_{0}^{2}$ ne s'annule pas dans $\Pi\left(\Lambda_{2, r}^{ \pm}\right)$.

Démonstration. - On a $u^{1}=V_{1}\left(\begin{array}{c}\omega_{11} \\ 0\end{array}\right)$ et $u^{2}=V_{2}\left(\begin{array}{c}0 \\ \omega_{22}\end{array}\right)$; donc

$$
u_{0}^{1}=\omega_{0}^{1}\left(\begin{array}{c}
\partial_{x_{2}} \phi_{1} \\
-\partial_{x_{1}} \phi_{1}
\end{array}\right) \quad \text { et } \quad u_{0}^{2}=\omega_{0}^{2}\left(\begin{array}{c}
\partial_{x_{1}} \phi_{2} \\
\partial_{x_{2}} \phi_{2}
\end{array}\right) \text {. }
$$

Puisque $\nabla \phi_{j}$ ne s'annule pas, il suffit de vérifier que $\omega_{0}^{j}$ ne s'annule pas. De plus $\nabla \phi_{j} \cdot \partial_{x} \omega_{0}^{j}=0$, donc $\omega_{0}^{j}$ est constant sur les courbes de $\nabla \phi_{j}$. Il suffit donc de montrer que $\omega_{0}^{1}(0, \theta)$ et $\omega_{0}^{2}(0, \theta)$ ne s'annulent pas dans la bande $B_{a}$. Pour $r=0$ on a

$$
\left(\begin{array}{c}
\omega_{0}^{1} \\
\omega_{0}^{2}
\end{array}\right)=A^{-1}\left(\begin{array}{c}
v_{0}^{1} \\
v_{0}^{2}
\end{array}\right)=A^{-1} T(\theta, 0)\left(\begin{array}{c}
r_{1,0} \\
0
\end{array}\right)
$$

et puisque

$$
A^{-1}=\frac{1}{\operatorname{det} A}\left(\begin{array}{cc}
\partial_{x_{2}} \phi_{2} & -\partial_{x_{1}} \phi_{2} \\
\partial_{x_{1}} \phi_{1} & \partial_{x_{2}} \phi_{1}
\end{array}\right),
$$

on a

$$
\begin{aligned}
\omega_{0}^{1} & =\frac{1}{\operatorname{det} A} r_{1,0}\left(\partial_{x_{2}} \phi_{2}-\partial_{x_{1}} \phi_{2}\right) \cdot T(\theta, 0)\left(\begin{array}{l}
1 \\
0
\end{array}\right), \\
\omega_{0}^{2} & =\frac{1}{\operatorname{det} A} r_{1,0}\left(\partial_{x_{1}} \phi_{1} \partial_{x_{2}} \phi_{1}\right) \cdot T(\theta, 0)\left(\begin{array}{l}
1 \\
0
\end{array}\right) .
\end{aligned}
$$

BULLETIN DE LA SOCIÉTÉ MATHÉMATIQUE DE FRANCE 
$T(\theta, 0)\left(\begin{array}{l}1 \\ 0\end{array}\right)$ engendre le sous-espace propre associé à la valeur propre $a_{1}^{\psi}(\theta, 0)$ $(=0)$ de la matrice $n_{0}^{\psi}(\theta, 0)$, c'est-à-dire le noyau de $n_{0}\left(\theta, \pm c_{R}^{-1} R(\theta)\right)=n_{0 \mid \Sigma^{ \pm}}$.

Or $r_{1,0}$ ne s'annule pas dans $B_{a}$, donc d'après (19)

$$
\omega_{0}^{1}(0, \theta)=0 \Longleftrightarrow n_{0 \mid \Sigma^{ \pm}} \nabla \phi_{2}^{ \pm}(0, \theta)=0,
$$

et d'après (20)

$$
\omega_{0}^{2}(0, \theta)=0 \Longleftrightarrow n_{0 \mid \Sigma^{ \pm}}\left(\begin{array}{c}
\partial_{x_{2}} \phi_{1}^{ \pm}(0, \theta) \\
-\partial_{x_{1}} \phi_{1}^{ \pm}(0, \theta)
\end{array}\right)=0 .
$$

Il suffit de faire le calcul pour s'apercevoir que

$$
\begin{gathered}
n_{0 \mid \Sigma^{ \pm}} \nabla \phi_{2}^{ \pm}(0, \theta)=0 \Longleftrightarrow c_{R}^{2}=c_{1}^{2}+c_{2}^{2}, \\
n_{0 \mid \Sigma^{ \pm}}\left(\begin{array}{c}
\partial_{x_{2}} \phi_{1}^{ \pm}(0, \theta) \\
-\partial_{x_{1}} \phi_{1}^{ \pm}(0, \theta)
\end{array}\right)=0 \Longleftrightarrow c_{R}^{2}=c_{1}^{2}+c_{2}^{2} .
\end{gathered}
$$

Or la relation $c_{R}^{2}=c_{1}^{2}+c_{2}^{2}$ n'est jamais vérifiée puisque $c_{R}<c_{1}<c_{2}$, d'où le résultat.

4.5. Conclusion. - Soit $u^{1}$ et $u^{2}$ des réalisations des symboles analytiques construits dans la proposition 4.5. Il existe une constante $C_{1}>0$ telle que

$$
\begin{aligned}
& Q(h) u^{1} \exp \left(i \phi_{1} / h\right)=O\left(\exp \left(-\left(C_{1}+\operatorname{Im} \phi_{1}\right) / h\right)\right), \\
& Q(h) u^{2} \exp \left(i \phi_{2} / h\right)=O\left(\exp \left(-\left(C_{1}+\operatorname{Im} \phi_{2}\right) / h\right)\right) .
\end{aligned}
$$

Donc si l'on pose $u=u^{1} \exp \left(i \phi_{1} / h\right)+u^{2} \exp \left(i \phi_{2} / h\right)$, on a

$$
Q(h) u=O\left(\exp \left(-\left(C_{1}+\min \left(\operatorname{Im} \phi_{1}, \operatorname{Im} \phi_{2}\right)\right) / h\right)\right) .
$$

Si $u^{1}$ et $u^{2}$ désignent les symboles analytiques construits dans la proposition 4.5,

$$
\exp (-i \psi / h) B\left(u^{1} \exp \left(i \phi_{1} / h\right)+u^{2} \exp \left(i \phi_{2} / h\right)\right)_{\mid r=0}
$$

est un symbole analytique, qui est nul d'après les propositions 4.3 et 4.5 . Il existe donc $C_{2}>0$ tel que

$$
B u_{\mid r=0}=O\left(\exp \left(-\left(C_{2}+\operatorname{Im} \psi\right) / h\right)\right) .
$$

Choisissons $\psi$ telle que $\operatorname{Im} \psi(\theta)=0$ pour $\theta$ dans $\mathbb{R}$. C'est possible car $\operatorname{Im} \psi$ est constante sur $\mathbb{R}$. Il existe alors une constante $C>0$ telle que

$$
\begin{cases}Q(h) u=O\left(\exp \left(-\left(C+\min \left(\operatorname{Im} \phi_{1}, \operatorname{Im} \phi_{2}\right)\right) / h\right)\right) & \text { dans } \Pi\left(\Lambda_{1, r}^{ \pm}\right) \cap \Pi\left(\Lambda_{2, r}^{ \pm}\right), \\ B u_{\mid r=0}=O(\exp (-C / h)) & \text { pour } \theta \in \mathbb{R} .\end{cases}
$$

TOME $132-2004-\mathrm{N}^{\mathrm{O}} 2$ 


\section{Condition de quantification}

Nous allons tout d'abord montrer des résultats sur les symboles analytiques ne dépendant que de $h$ :

Proposition 5.1. - (i) Si a $(z)$ est une fonction analytique près de $b_{0}$ et $b(h)=\sum_{j \geq 0} b_{j} h^{j}$ est un symbole analytique, alors $a(b(h))$ est un symbole analytique.

(ii) Si $a(h)$ est un symbole analytique et $b(h)$ est un symbole analytique d'ordre -1 , alors $a(b(h))$ est un symbole analytique.

(iii) Si $a(h)=\sum_{j \geq 1} a_{j} h^{j}$ est un symbole analytique d'ordre -1 vérifiant $a_{1} \neq 0$, l'unique symbole formel d'ordre $-1 b(h)$ vérifiant $a(b(h))=h$ est un symbole analytique.

On a besoin du lemme suivant :

Lemme 5.2. - Soient $k, n_{1}, \ldots, n_{k}$ des entiers strictement positifs. On a :

$$
k^{k} \prod_{i=1}^{k} n_{i}^{n_{i}} \leq\left(\sum_{i=1}^{k} n_{i}\right)^{\sum_{i=1}^{k} n_{i}}
$$

Démonstration. - On va montrer cela par récurrence sur $j=\sum_{i=1}^{k} n_{i}$. Remarquons que $1 \leq k \leq j$. On note $P(j)$ la propriété suivante :

$$
\forall k \in\{1, \ldots, j\}, \forall\left(n_{1}, \ldots, n_{k}\right) \in\left(\mathbb{N}^{*}\right)^{k} \text { t.q. } \sum_{i=1}^{k} n_{i}=j, \quad k^{k} \prod_{i=1}^{k} n_{i}^{n_{i}} \leq j^{j} .
$$

$P(1)$ est évidemment vraie. Pour $j=2$, si $k=1$ c'est évident car $n_{1}=j$, et si $k=2$ c'est vrai aussi car $k=j$ et $n_{1}=n_{2}=1$. Donc $P(2)$ est aussi vraie.

Soit maintenant $j \geq 3$ et supposons que $P(n)$ est vraie pour $n=1, \ldots, j-1$. On va montrer que $P(j)$ est vraie. Si $k=1$ c'est évident car $n_{1}=j$. Si $k=j$ c'est évident aussi car $n_{1}=\cdots=n_{k}=1$. Supposons maintenant que $2 \leq k \leq j-1$. Puisque $n_{1} \geq 1, P\left(j-n_{1}\right)$ est vraie, on obtient

$$
k^{k} \prod_{i=1}^{k} n_{i}^{n_{i}} \leq k^{k} n_{1}^{n_{1}} \frac{\left(j-n_{1}\right)^{j-n_{1}}}{(k-1)^{k-1}}
$$

Il suffit d'étudier la fonction $x \mapsto x \ln x+(j-x) \ln (j-x)$ sur l'intervalle $[1, j-1]$ pour se rendre compte qu'elle atteint son maximum aux extrémités de l'intervalle. On a donc

$$
n_{1}^{n_{1}}\left(j-n_{1}\right)^{j-n_{1}} \leq(j-1)^{j-1}
$$

BULletin DE LA SOCiÉtÉ MATHÉMATIQUE DE FRANCE 
et avec (22) on obtient

$$
\begin{aligned}
k^{k} \prod_{i=1}^{k} n_{i}^{n_{i}} & \leq \frac{k^{k}(j-1)^{j-1}}{(k-1)^{k-1} j^{j}} j^{j} \leq\left(\frac{k}{k-1}\right)^{k-1}\left(\frac{j-1}{j}\right)^{j} \frac{k}{j-1} j^{j} \\
& \leq\left(\frac{k}{k-1}\right)^{k-1}\left(\frac{j-1}{j}\right)^{j} j^{j} .
\end{aligned}
$$

Or $k \mapsto(k /(k-1))^{k-1}$ est croissante et tend vers $e$ lorsque $k$ tend vers $+\infty$ et $j \mapsto((j-1) / j)^{j}$ est croissante et tend vers $1 / e$ quand $j$ tend vers $+\infty$. On en déduit avec (23) que

$$
k^{k} \prod_{i=1}^{k} n_{i}^{n_{i}} \leq e \frac{1}{e} j^{j} \leq j^{j} .
$$

Donc $P(j)$ est vraie.

Preuve de la proposition 5.1. - Les symboles ne dépendant que de $h$, on va définir les pseudonormes suivantes : soit $a(h)=\sum_{j \geq 0} a_{j} h^{j}$ un symbole et $C_{j}(a)$ le plus petit nombre vérifiant $\left|a_{j}\right| \leq C_{j}(a) j^{j}$, on définit

$$
\|a\|_{\mu}=\sum_{k=0}^{+\infty} C_{k}(a) \mu^{k}
$$

Alors $a(h)$ est un symbole analytique si et seulement si il existe $\mu>0$ tel que $\|a\|_{\mu}<+\infty$. On a aussi $\|a \cdot b\|_{\mu}=\|a \sharp b\|_{\mu} \leq\|a\|_{\mu} \cdot\|b\|_{\mu}$.

(i) $a(h)$ étant analytique au voisinage de $b_{0}$, on a $a(z)=\sum_{j=0}^{+\infty} a_{j}\left(z-b_{0}\right)^{j}$ et il existe $C>0$ telle que $\left|a_{j}\right| \leq C^{j} . b(h)-b_{0}$ étant un symbole analytique d'ordre $-1,\left\|b-b_{0}\right\|_{\mu}=O(\mu)$, donc si $\mu>0$ est assez petit on a $\left\|b-b_{0}\right\|_{\mu} \leq 1 / 2 C$. On a alors

$$
\|a \circ b\|_{\mu} \leq \sum_{k=0}^{+\infty} a_{k}\left\|b-b_{0}\right\|_{\mu}^{k} \leq 2<+\infty .
$$

Le symbole $a \circ b$ est donc bien analytique.

(ii) On a $a(b(h))=\sum_{k \geq 0} a_{k}\left(\sum_{\ell \geq 1} b_{\ell} h^{\ell}\right)^{k}=\sum_{j \geq 0} d_{j} h^{j}$ où $d_{0}=a_{0}$ et pour $j \geq 1$ on a

$$
d_{j}=\sum_{k=1}^{j} a_{k} \sum_{n_{1}+\cdots+n_{k}=j} b_{n_{1}} \cdots b_{n_{k}} .
$$

TOME $132-2004-\mathrm{N}^{\mathrm{O}} 2$ 
D'après le lemme 5.2 on obtient

$$
\begin{aligned}
\left|d_{j}\right| & \leq \sum_{k=1}^{j} C_{k}(a) k^{k} \sum_{n_{1}+\cdots+n_{k}=j} C_{n_{1}}(b) \cdots C_{n_{k}}(b) n_{1}^{n_{1}} \cdots n_{k}^{n_{k}} \\
& \leq\left(\sum_{k=1}^{j} C_{k}(a) \sum_{n_{1}+\cdots+n_{k}=j} C_{n_{1}}(b) \cdots C_{n_{k}}(b)\right) j^{j} .
\end{aligned}
$$

On en déduit

$$
C_{j}(a \circ b) \leq \sum_{k=1}^{j} C_{k}(a) \sum_{n_{1}+\cdots+n_{k}=j} C_{n_{1}}(b) \cdots C_{n_{k}}(b)
$$

et donc

$$
\|a \circ b\|_{\mu}=\sum_{j=0}^{+\infty} C_{j}(a \circ b) \mu^{j} \leq \sum_{k=0}^{+\infty} C_{k}(a)\left(\sum_{\ell=1}^{+\infty} C_{\ell}(b) \mu^{\ell}\right)^{k} .
$$

$a(h)$ étant un symbole analytique, $\sum_{k \geq 0} C_{k}(a) \mu^{k}$ converge pour $\mu<\mu_{a}$ donc est analytique en $\mu$ au voisinage de 0 . De même $\sum_{\ell>1} C_{\ell}(b) \mu^{\ell}$ converge pour $\mu<\mu_{b}$ donc est analytique au voisinage de 0 , et de plus vaut 0 en 0 . Par composition des séries entières on obtient que le membre de droite de (24) converge pour $\mu$ assez petit et donc $\|a \circ b\|_{\mu}<+\infty$. Le symbole $a \circ b$ est donc bien un symbole analytique.

(iii) $a(h)=\sum_{j \geq 1} a_{j} h^{j}$ étant un symbole analytique il existe $C>0$ tel que $\left|a_{j}\right| \leq C^{j+1} j^{j}$. On cherche $b(h)=\sum_{j \geq 1} b_{j} h^{j}$ tel que

$$
a(b(h))=\sum_{k \geq 1} a_{k}\left(\sum_{n \geq 1} b_{n} h^{n}\right)^{k}=h
$$

(somme finie en chaque degré d'homogénéité). Les nombres $b_{j}$ sont déterminés de façon unique par les équations suivantes :

$$
a_{1} b_{1}=1 \quad \text { et } \quad \forall j \geq 2, \quad a_{1} b_{j}+\sum_{k=2}^{j} a_{k} \sum_{n_{1}+\cdots+n_{k}=j} b_{n_{1}} \cdots b_{n_{k}}=0 .
$$

On va d'abord montrer par récurrence que pour $j \geq 2$

$$
b_{j}=\frac{1}{a_{1}^{2 j-1}} \sum_{n_{1}+\cdots+n_{j-1}=2 j-2} \alpha\left(n_{1}, \ldots, n_{j-1}\right) a_{n_{1}} \cdots a_{n_{j-1}}
$$

où les $\alpha\left(n_{1}, \ldots, n_{j-1}\right)$ sont des nombres entiers. On a $b_{2}=-a_{2} / a_{1}^{3} ;$ donc la propriété est vraie pour $j=2$ avec $n_{1}=2$ et $\alpha\left(n_{1}\right)=-1$. Soit $j \geq 3$, supposons 
que la propriété soit vraie pour $1, \ldots, j-1$. D'après $(25)$

$$
\begin{aligned}
b_{j} & =\sum_{k=2}^{j} \sum_{n_{1}+\cdots+n_{k}=j}-\frac{a_{k} a_{1}^{k-2}}{a_{1}^{k-1}} b_{n_{1}} \cdots b_{n_{k}} \\
& =-\frac{1}{a_{1}^{2 j-1}} \sum_{k=2}^{j} \sum_{n_{1}+\cdots+n_{k}=j} a_{k} a_{1}^{k-2} b_{n_{1}} a_{1}^{2 n_{1}-1} \cdots b_{n_{k}} a_{1}^{2 n_{k}-1} .
\end{aligned}
$$

D'après l'hypothèse de récurrence et le fait que $b_{1}=1 / a_{1}$, dans la somme précédente chacun des termes $a_{k} a_{1}^{k-2} b_{n_{1}} a_{1}^{2 n_{1}-1} \cdots b_{n_{k}} a_{1}^{2 n_{k}-1}$ est de la forme

$$
\sum \beta\left(i_{1}, \ldots, i_{m}\right) a_{i_{1}} \cdots a_{i_{m}}
$$

où $m=1+k-2+n_{1}-1+\cdots+n_{k}-1=j-1$ et

$$
i_{1}+\cdots+i_{m}=k+k-2+2 n_{1}-2+\cdots+2 n_{k}-2=2 j-2 .
$$

La propriété $(26)$ est donc vraie pour tout $j \geq 2$.

On peut supposer sans perte de généralité que $a_{1}>0$. Remarquons que lorsque $a_{1}$ est fixé $\left|b_{j}\right|$ est maximal lorsque $a_{k}=-C^{k+1} k^{k}$ pour $k \geq 2$. Il suffit donc de montrer (iii) dans le cas où $a(h)=a_{1} h+\sum_{k \geq 2}-C^{k+1} k^{k} h^{k}$. Dans ce cas, dans (26) chacun des termes est positif.

D'après le lemme 5.2 , si $n_{1}+\cdots+n_{j-1}=2 j-2$ on a

$$
n_{1}^{n_{1}} \cdots n_{j-1}^{n_{j-1}} \leq \frac{(2 j-2)^{2 j-2}}{(j-1)^{j-1}} \leq 2^{2 j-2}(j-1)^{j-1} \leq 4^{j} j^{j} .
$$

Posons maintenant $c_{j}=a_{j} / j^{j}$. D'après (26) et (27) on a

$$
b_{j} \leq 4^{j} j^{j} \underbrace{\frac{1}{c_{1}^{2 j-1}} \sum_{n_{1}+\cdots+n_{j-1}=2 j-2} \alpha\left(n_{1}, \ldots, n_{j-1}\right) c_{n_{1}} \cdots c_{n_{j-1}}}_{:=d_{j}} .
$$

Pour $j \geq 2, c_{j}=-C^{j+1} ; c(h)=\sum_{j \geq 1} c_{j} h^{j}$ est donc une fonction analytique au voisinage de 0 et $d(h)=\sum_{j \geq 1} d_{j} h^{j}$, inverse de $c(h)$ pour la composition, est donc analytique au voisinage de 0 . Il existe donc $C^{\prime}>0$ telle que $d_{j} \leq C^{\prime j}$ et finalement on obtient $0<b_{j} \leq\left(4 C^{\prime}\right)^{j} j^{j}$. $b(h)$ est donc un symbole analytique.

Remarque. - Dans la démonstration de (iii), nous n'avons pas utilisé les pseudonormes pour la raison suivante. On peut exprimer le symbole inverse comme somme d'une série dont les termes sont des itérés pour la composition o de symboles analytiques, mais il n'est pas évident que tous les itérés ont leur pseudonorme finie pour un même $\mu$.

Proposition 5.3. - Le symbole analytique classique $c(h)$ (voir la proposition 4.4) vérifie $c(h) \cdot \overline{c(h)}=1$.

TOME $132-2004-\mathrm{N}^{\mathrm{O}} 2$ 
Démonstration. - Soit $a$ un réel tel que $0<a<\pi$. Soit $\chi=\chi(\theta)$ à support compact telle que :

- $\chi=0$ sur $]-\infty,-a] \cup[2 \pi+a,+\infty[;$

- $\chi=1$ sur $[a, 2 \pi-a] ; 0 \leq \chi \leq 1 ; \chi>0$ sur $]-a, a[$;

- $\chi(-\theta)-\frac{1}{2}=\frac{1}{2}-\chi(\theta)$ pour tout $\theta$ dans $[-a, a]$;

- $\chi(\pi+\theta)=\chi(\pi-\theta)$ pour tout $\theta$ dans $\mathbb{R}$.

On a alors $1-\chi(2 \pi+\theta)=1-\chi(-\theta)=\chi(\theta)$ pour tout $\theta$ dans $[-a, a]$. On peut choisir $\chi_{1}=\chi_{1}(\theta)$ et $\chi_{2}=\chi_{2}(\theta)$ à supports compacts vérifiant les conditions suivantes :

- $0 \leq \chi_{1} \leq 1 ; \chi_{1}=1$ sur $[-a, a]$

- $\left.\operatorname{Supp} \chi_{1} \subset\right]-\pi, \pi\left[; 0 \leq \chi_{2} \leq 1\right.$;

- $\chi_{2}(\theta)=\chi_{1}(\theta-2 \pi)$ pour tout $\theta$ dans $\mathbb{R}$.

On a alors $\chi_{2}=1 \operatorname{sur}[2 \pi-a, 2 \pi+a]$, Supp $\left.\chi_{2} \subset\right] \pi, 3 \pi\left[\right.$ et $\operatorname{Supp} \chi_{1} \cap \operatorname{Supp} \chi_{2}=\varnothing$.

Dans la suite $(.,$.$) désigne le produit scalaire usuel dans L^{2}(\mathbb{R})$ et $[.,$.$] le$ commutateur de deux opérateurs.

D'après le travail de Sjöstrand-Vodev [10], $N_{e}(h)$ est auto-adjoint pour $h$ réel. $N_{e}^{\psi}(h)$ est donc aussi un opérateur $h$-pseudodifférentiel analytique, autoadjoint pour $h$ réel. D'après la proposition 4.3, on a donc

$$
\begin{aligned}
\left(\left[N_{e}^{\psi}(h), \chi\right] v, v\right) & =\left(N_{e}^{\psi}(h) \chi v, v\right)-\left(\chi N_{e}^{\psi}(h) v, v\right) \\
& =\left(\chi v, N_{e}^{\psi}(h) v\right)-\left(\chi N_{e}^{\psi}(h) v, v\right)=0 .
\end{aligned}
$$

On a $\chi_{1}+\chi_{2}=1$ sur le support de $\partial_{\theta}^{\alpha} \chi$ pour tout $\alpha \geq 1$, d'où

$$
\left[N_{e}^{\psi}(h), \chi\right] \cdot\left(1-\chi_{1}-\chi_{2}\right)=0 .
$$

Or

$$
\begin{aligned}
\left(\left[N_{e}^{\psi}(h), \chi\right] \chi_{2} v, v\right) & =-\left(\left[N_{e}^{\psi}(h), 1-\chi\right] \chi_{2} v, v\right) \\
& =-\left(\left[N_{e}^{\psi}(h), \chi(.-2 \pi)\right] \chi_{1}(.-2 \pi) v, v\right) \\
& =-\left(\left[N_{e}^{\psi}(h), \chi\right] \chi_{1} v(.+2 \pi), v(.+2 \pi)\right) \\
& =-|c(h)|^{2}\left(\left[N_{e}^{\psi}(h), \chi\right] \chi_{1} v, v\right) .
\end{aligned}
$$

D'après (28), (29) et (30), on obtient

$$
\left(1-|c(h)|^{2}\right) \underbrace{\left(\left[N_{e}^{\psi}(h), \chi\right] \chi_{1} v, v\right)}_{:=\mathcal{I}}=0 .
$$

Il reste à montrer que $\mathcal{I}$ n'est pas nulle au sens des symboles analytiques. Soient $n_{0}(\theta, \eta)$ et $n_{0}^{\psi}(\theta, \eta)$ les symboles principaux respectifs des opérateurs $N_{e}(h)$ et $N_{e}^{\psi}(h)$. On a $n_{0}^{\psi}(\theta, \eta)=n_{0}\left(\theta, \eta+\partial_{\theta} \psi(\theta)\right)=n_{0}\left(\theta, \eta \pm c_{R}^{-1} R(\theta)\right)$. Puisque $N_{e}(h)$ est auto-adjoint, $n_{0}(\theta, \eta)$ est une matrice hermitienne et on a

$$
n_{0}(\theta, \eta)=\widetilde{a}_{1}(\theta, \eta)\left(c_{R}^{2} \eta^{2}-R(\theta)^{2}\right) \Pi_{1}(\theta, \eta)+a_{2}(\theta, \eta) \Pi_{2}(\theta, \eta),
$$

BUlletin DE LA SOCiÉtÉ MATHÉmATiQUe DE FRANCE 
où $\Pi_{1}$ et $\Pi_{2}$ sont les projecteurs orthogonaux auto-adjoints associés aux valeurs propres $a_{1}(\theta, \eta)$ et $a_{2}(\theta, \eta)$ de $n_{0}$, et $\widetilde{a}_{1}(\theta, \eta)$ et $a_{2}(\theta, \eta)$ sont différents de 0 pour $(\theta, \eta)$ dans un voisinage de $\Sigma$ (voir $(5))$.

Le symbole principal de $\left[N_{e}^{\psi}(h), \chi\right] \chi_{1}$ est $h / i \partial_{\eta} n_{0}^{\psi} \partial_{\theta} \chi \cdot \chi_{1}$, donc le premier terme dans le développement de $\mathcal{I}$ est

$$
\mathcal{I}_{0}:=\frac{h}{i} \int\left(\partial_{\eta} n_{0}^{\psi}(\theta, 0) \partial_{\theta} \chi(\theta) \cdot \chi_{1}(\theta) v_{0}(\theta) \mid v_{0}(\theta)\right) \mathrm{d} \theta
$$

où $\left(. \mid\right.$.) désigne le produit hermitien usuel dans $\mathbb{C}^{2}$. On sait que

$$
v_{0}(\theta)=T(\theta, 0) U_{0}(\theta, 0)\left(\begin{array}{c}
r_{1,0}(\theta) \\
0
\end{array}\right)=T(\theta, 0)\left(\begin{array}{l}
1 \\
0
\end{array}\right) r_{1,0}(\theta) .
$$

Or $T(\theta, 0)\left(\begin{array}{l}1 \\ 0\end{array}\right)$ engendre le sous-espace propre de $n_{0}^{\psi}(\theta, 0)$ associé à la valeur propre $a_{1}^{\psi}(\theta, 0)=0$, c'est-à-dire $\operatorname{Im} \Pi_{1}\left(\theta, \pm c_{R}^{-1} R(\theta)\right)$. Donc

$$
v_{0} \in \operatorname{Im} \Pi_{1}\left(\theta, \pm c_{R}^{-1} R(\theta)\right)=\operatorname{ker} \Pi_{2}\left(\theta, \pm c_{R}^{-1} R(\theta)\right) .
$$

On a

$$
\begin{aligned}
& \partial_{\eta} n_{0}^{\psi}(\theta, 0)= \partial_{\eta} n_{0}\left(\theta, \pm c_{R}^{-1} R(\theta)\right) \\
&= \pm 2 c_{R} R(\theta) \widetilde{a}_{1}\left(\theta, \pm c_{R}^{-1} R(\theta)\right) \Pi_{1}\left(\theta, \pm c_{R}^{-1} R(\theta)\right) \\
& \quad+\partial_{\eta} a_{2}\left(\theta, \pm c_{R}^{-1} R(\theta)\right) \Pi_{2}\left(\theta, \pm c_{R}^{-1} R(\theta)\right) \\
& \quad+a_{2}\left(\theta, \pm c_{R}^{-1} R(\theta)\right) \partial_{\eta} \Pi_{2}\left(\theta, \pm c_{R}^{-1} R(\theta)\right) .
\end{aligned}
$$

De plus $\Pi_{1} \cdot \Pi_{2}=\Pi_{2} \cdot \Pi_{1}=0$; donc $\partial_{\eta} \Pi_{2} \cdot \Pi_{1}+\Pi_{2} \cdot \partial_{\eta} \Pi_{1}=0$ et en composant à gauche par $\Pi_{1}$ on obtient $\Pi_{1} \cdot \partial_{\eta} \Pi_{2} \cdot \Pi_{1}=0$. D'après $(32)$, on obtient

$$
\begin{aligned}
\Pi_{1}(\theta, & \left. \pm c_{R}^{-1} R(\theta)\right) \partial_{\eta} n_{0}^{\psi}(\theta, 0) \Pi_{1}\left(\theta, \pm c_{R}^{-1} R(\theta)\right) \\
& = \pm 2 c_{R} R(\theta) \widetilde{a}_{1}\left(\theta, \pm c_{R}^{-1} R(\theta)\right) \Pi_{1}\left(\theta, \pm c_{R}^{-1} R(\theta)\right) .
\end{aligned}
$$

D'après $(31)$ et $(33), \mathcal{I}_{0}$ est égal à

$$
\begin{aligned}
& \frac{h}{i} \int\left(\partial_{\eta} n_{0}^{\psi}(\theta, 0) \partial_{\theta} \chi(\theta) \cdot \chi_{1}(\theta) \Pi_{1}\left(\theta, \pm c_{R}^{-1} R(\theta)\right) v_{0}(\theta) \mid\right. \\
&\left.\Pi_{1}\left(\theta, \pm c_{R}^{-1} R(\theta)\right) v_{0}(\theta)\right) \mathrm{d} \theta \frac{h}{i} \int\left(\Pi_{1}\left(\theta, \pm c_{R}^{-1} R(\theta)\right) \partial_{\eta} n_{0}^{\psi}(\theta, 0)\right. \\
&\left.\times \Pi_{1}\left(\theta, \pm c_{R}^{-1} R(\theta)\right) \partial_{\theta} \chi(\theta) \cdot \chi_{1}(\theta) v_{0}(\theta) \mid v_{0}(\theta)\right) \mathrm{d} \theta \\
&= \pm 2 c_{R} \frac{h}{i} \int \widetilde{a}_{1}\left(\theta, \pm c_{R}^{-1} R(\theta)\right) \partial_{\theta} \chi(\theta) \cdot \chi_{1}(\theta)\left(v_{0} \mid v_{0}\right) R(\theta) \mathrm{d} \theta .
\end{aligned}
$$

On a $\widetilde{a}_{1}\left(\theta, \pm c_{R}^{-1} R(\theta)\right) \neq 0,\left(v_{0} \mid v_{0}\right)>0, R(\theta)>0, \partial_{\theta} \chi \geq 0$ sur le support de $\chi_{1}$ et $\chi_{1}(\theta)=1$ lorsque $\partial_{\theta} \chi(\theta)>0$, donc $\mathcal{I}_{0} \neq 0$. La proposition est démontrée. 
Soient $u^{1}$ et $u^{2}$ les symboles analytiques construits dans la proposition 4.5 ; on pose

$$
u=u^{1} \exp \left(i \phi_{1} / h\right)+u^{2} \exp \left(i \phi_{2} / h\right) .
$$

D'après la proposition 4.6 et la dernière partie de la proposition 3.2 , on a

$$
\left.u(r, \theta+2 \pi ; h)=c(h) \exp \left( \pm i c_{R}^{-1} \ell(\Gamma) / h\right)\right) u(r, \theta ; h) .
$$

$u(r, \theta ; h)$ est formellement $2 \pi$-périodique en la variable $\theta$ si

$$
c(h) \exp \left( \pm i c_{R}^{-1} \ell(\Gamma) / h\right)=1 .
$$

Puisque $c(h) \cdot \overline{c(h)}=1$, on a $\left|c_{0}\right|^{2}=1$. Soit $z \mapsto \ln z$ une détermination du logarithme près de $c_{0}$; posons $d(h)=\mp i \ln c(h)$. La fonction ln étant analytique près de $c_{0}$ et $c(h)$ étant un symbole analytique, $d(h)=\sum_{j \geq 0} d_{j} h^{j}$ est un symbole analytique classique d'après la proposition 5.1. De plus puisque $c(h) \cdot \overline{c(h)}=1$, les $d_{j}$ sont réels, et puisque $c^{-}(h)=\overline{c^{+}(h)}, d(h)$ est indépendant de \pm . On a alors $c^{ \pm}(h)=\exp ( \pm i d(h))$ et d'après $(34)$ on obtient la condition de quantification:

$$
d(h)+c_{R}^{-1} \ell(\Gamma) / h=k \cdot 2 \pi, \quad k \in \mathbb{Z} .
$$

Proposition 5.4. - Il existe un symbole analytique classique

$$
h\left(\frac{1}{k}\right)=\frac{\ell(\Gamma)}{2 \pi c_{R}} k^{-1}+\sum_{j \geq 2} e_{j} k^{-j},
$$

avec $e_{j} \in \mathbb{R}, k \in \mathbb{N}^{*}$, vérifiant la condition de quantification (35).

Démonstration. - Lorsque $k$ appartient à $\mathbb{Z}^{*},(35)$ devient $h=a(h) k^{-1}$ où

$$
a(h)=\frac{c_{R}^{-1} \ell(\Gamma)}{2 \pi}+\frac{1}{2 \pi} h \mathrm{~d}(h)
$$

est un symbole analytique classique elliptique d'ordre $0 . a(h)$ admet donc un symbole analytique classique inverse (pour le produit) $b(h)=\sum_{j \geq 0} b_{j} h^{j}$ avec $b_{0}=2 \pi c_{R} / \ell(\Gamma)$. On obtient alors $h b(h)=1 / k$ et d'après la proposition 5.1, $h b(h)$ admet un inverse (pour la composition $\circ) e(h)=\sum_{j \geq 1} e_{j} h^{j}$ qui est un symbole analytique vérifiant $e_{1}=\ell(\Gamma) / 2 \pi c_{R}$. On obtient alors $h=\sum_{j \geq 1} e_{j} k^{-j}$.

Donc si $h_{k}$ est une réalisation du symbole analytique $h\left(k^{-1}\right)=\sum_{j \geq 1} e_{j} k^{-j}$, il existe une constante $C>0$ telle que pour $h$ dans $\left\{h_{k} ; k \in \mathbb{N}^{*}\right\}$ on ait

$$
\begin{aligned}
u(r, \theta+ & 2 \pi ; h)-u(r, \theta ; h) \\
& =O\left(\exp \left(-\left(C+\min \left(\operatorname{Im} \phi_{1}(r, \theta), \operatorname{Im} \phi_{2}(r, \theta)\right)\right) / h\right)\right) .
\end{aligned}
$$

BULlETiN DE LA SOCIÉtÉ MATHÉMATIQUE DE FRANCE 


\section{Localisation des résonances de Rayleigh}

En tronquant (en la variable $r$ ) près du bord $\Gamma$ les solutions BKW construites dans la section 4 , on obtient des fonctions presque $2 \pi$-périodiques pour $h$ dans $\left\{h_{k} ; k \in \mathbb{N}^{*}\right\}$ et analytiques en la variable $\theta$. En leur rajoutant un terme exponentiellement petit à support compact on obtient des quasimodes $W^{ \pm}(h)$ exactement $2 \pi$-périodiques (pour $h$ dans $\left\{h_{k} ; k \in \mathbb{N}^{*}\right\}$ ), à supports compacts, presque orthogonaux entre eux pour la norme $L^{2}(\Omega)$ et vérifiant exactement la condition de Neumann au bord (voir la proposition 6.4).

6.1. Résolution de l'équation $f(z+T)-f(z)=g(z)$. - Nous allons d'abord rappeler un vieux résultat (voir [2]) qui donne sous forme intégrale une solution particulière de l'équation d'inconnue $f$

$$
f(z+T)-f(z)=g(z),
$$

où $T$ est un nombre strictement positif fixé et $g$ est analytique. Nous montrerons ensuite que si $g(z ; h)$ est exponentiellement petit par rapport à $h$, alors l'une des solutions $f(z ; h)$, celle justement donnée sous forme intégrale, l'est aussi.

- Si $g=0$ alors $f$ est $T$-périodique. Les solutions du problème homogène sont donc connues.

- Si $g \neq 0$ est analytique dans la bande $|\operatorname{Im} z|<a$ où $a>0$, alors on fixe $\delta>0$ et pour $|\operatorname{Im} z|<a-\delta$, on considère

$$
(I g)(z)=\frac{i}{2 T} \int_{\gamma} \operatorname{cotan}\left(\frac{\pi}{T}\left(z-z^{\prime}+0\right)\right) g\left(z^{\prime}\right) \mathrm{d} z^{\prime} .
$$

où $\gamma$ est un chemin qui joint le point $-i(a-\delta)$ au point $i(a-\delta)$ et qui passe par $z$. On peut par exemple choisir le segment de droite joignant $-i(a-\delta)$ à $z$ augmenté du segment de droite joignant $z$ à $i(a-\delta)$. On intègre donc sur un chemin juste à gauche de $z$; c'est bien défini par déformation de contour et analyticité de l'intégrande.

La fonction $I g$ est analytique en la variable $z$. Fixons $z_{0}$; si on choisit un chemin $\gamma_{0}$ juste à gauche de $z_{0}$ dans un voisinage de $z_{0}, I g$ est définie par intégration le long de $\gamma_{0}$. Alors $I g$ est analytique au voisinage de $z_{0}$ car on intègre alors une fonction analytique en le paramètre $z$ sur un chemin fixe $\gamma_{0}$ sur lequel l'intégrande est parfaitement contrôlé.

La fonction $z \mapsto \operatorname{cotan}\left(\pi / T\left(z-z^{\prime}+0\right)\right)$ étant $T$-périodique, on obtient

$$
(I g)(z+T)-(I g)(z)=\frac{i}{2 T} \int_{\tilde{\gamma}} \operatorname{cotan}\left(\frac{\pi}{T}\left(z-z^{\prime}+0\right)\right) g\left(z^{\prime}\right) \mathrm{d} z^{\prime}
$$

où $\widetilde{\gamma}$ est un chemin fermé orienté trigonométriquement et entourant le point $z$. La fonction $z^{\prime} \mapsto \operatorname{cotan}\left(\pi / T\left(z-z^{\prime}+0\right)\right)$ a un unique pôle dans $\widetilde{\gamma}$ en $z^{\prime}=z$ d'ordre 1 et de résidu $-T / \pi$. Par la formule de Cauchy, on obtient donc

$$
(I g)(z+T)-(I g)(z)=g(z) .
$$

TOME $132-2004-\mathrm{N}^{\mathrm{O}} 2$ 
On obtient donc une solution particulière du problème non homogène qui est analytique dans la bande $|\operatorname{Im} z|<a-\delta$.

On a la propriété suivante :

LEMME 6.1. - Soient $B_{a}$ la bande $|\operatorname{Im} z|<a$ et $B_{a-\delta}$ la bande $|\operatorname{Im} z|<a-\delta$. Si $g=g(z ; h)$ est un élément de $H_{0}^{\text {loc }}\left(B_{a}\right)$ et $g \sim 0$, alors $(I g)(z ; h)$ est un élément de $H_{0}^{\mathrm{loc}}\left(B_{a-\delta}\right)$ vérifiant $I g \sim 0$.

Démonstration. - Comme $g$ est analytique dans $B_{a}$, alors $I g$ est analytique dans $B_{a-\delta}$. Soit un compact $K \subset B_{a-\delta}$; par déformation de contour, $I g$ peut être définie par intégration le long de $\gamma_{z}$ où $\gamma_{z}$ est le segment de droite joignant $-i(a-\delta)$ à $z-\frac{1}{2} T$ augmenté du segment de droite joignant $z-\frac{1}{2} T$ à $i(a-\delta)$.

Puisque $K$ est un compact inclus dans $B_{a-\delta}$ et que $\gamma_{z}$ évite les points $z-n T$ pour tout $n$ dans $\mathbb{N}$, il existe une constante $\varrho>0$ telle que

$$
\forall z \in K, \forall z^{\prime} \in \gamma_{z}, \forall n \in \mathbb{N}, \quad\left|z^{\prime}-(z-n T)\right| \geq \varrho .
$$

On en déduit qu'il existe une constante $M>0$ telle que

$$
\forall z \in K, \forall z^{\prime} \in \gamma_{z}, \quad\left|\operatorname{cotan}\left(\frac{\pi}{T}\left(z-z^{\prime}\right)\right)\right| \leq M
$$

$K$ étant compact, il existe une constante $L>0$ vérifiant

$$
\forall z \in K, \quad \ell\left(\gamma_{z}\right) \leq L .
$$

La réunion des $\gamma_{z}$ pour $z$ dans $K$ est incluse dans un compact $K^{\prime} \subset B_{a}$. Il existe donc une constante $C_{K^{\prime}}>0$ et un nombre $\varepsilon>0$ tels que

$$
\forall z^{\prime} \in K^{\prime}, \quad\left|g\left(z^{\prime} ; h\right)\right| \leq C_{K^{\prime}} \exp \left(-\frac{\varepsilon}{h}\right) .
$$

On déduit de tout cela que

$$
\forall z \in K, \quad|(I g)(z ; h)| \leq L M C_{K^{\prime}} \exp \left(-\frac{\varepsilon}{h}\right) .
$$

D'où $I g \sim 0$.

6.2. Construction des quasimodes de Rayleigh. - Soient $u^{1}=u^{1 \pm}$ et $u^{2}=u^{2 \pm}$ des réalisations des symboles analytiques construits dans la proposition 4.5. Soit $\phi_{1}=\phi_{1}^{ \pm}$et $\phi_{2}=\phi_{2}^{ \pm}$les fonctions construites dans la proposition 3.2 et vérifiant

$$
\forall \theta \in \mathbb{R}, \quad \operatorname{Im} \phi_{1}(0, \theta)=\operatorname{Im} \phi_{2}(0, \theta)=0 \text { et } \operatorname{Re} \phi_{1}(0, \theta)=\operatorname{Re} \phi_{2}(0, \theta)
$$

On peut faire ce choix car $\phi_{j}^{ \pm}$est définie à une constante près dans la proposition $3.2, \operatorname{Im} \phi_{j}^{ \pm}(0, \theta)$ est constante pour $\theta$ dans $\mathbb{R}$ et $\partial_{\theta} \phi_{j}^{ \pm}(0, \theta)$ ne dépend pas de $j$. On pose

$$
u=u^{1} \exp \left(i \phi_{1} / h\right)+u^{2} \exp \left(i \phi_{2} / h\right)
$$

BULletin DE LA SOCiÉtÉ MATHÉmATiQUe DE FRANCE 
Lemme 6.2. - Soit $P=\left[0, r_{0}\right] \times[0,2 \pi]$ et a et $\varphi$ deux fonctions $\mathcal{C}^{\infty}$ dans $P$ vérifiant

$$
\varphi(0, \theta)=0, \quad \operatorname{Re} \varphi \leq 0, \quad \operatorname{Re} \varphi\left(r_{0}, \theta\right)<0, \quad \partial_{r} \varphi \neq 0 .
$$

Alors si l'on note $I(h)=\int_{P} a \exp (\varphi / h)$, on a

$$
I(h)=C h+O\left(h^{2}\right) \quad \text { avec } \quad C=-\int_{0}^{2 \pi} \frac{a(0, \theta)}{\partial_{r} \varphi(0, \theta)} \mathrm{d} \theta .
$$

Démonstration. - Il suffit d'appliquer la formule de Green. On obtient

$$
\begin{aligned}
I(h)=h \int_{P} \frac{a}{\partial_{r} \varphi} \partial_{r}(\exp (\varphi / h)) & \\
=h\{-\underbrace{\int_{P} \partial_{r}\left(\frac{a}{\partial_{r} \varphi}\right) \exp (\varphi / h)}_{:=\mathcal{I}}+ & +\underbrace{\int_{0}^{2 \pi} \frac{a\left(r_{0}, \theta\right)}{\partial_{r} \varphi\left(r_{0}, \theta\right)} \exp \left(\varphi\left(r_{0}, \theta\right) / h\right) \mathrm{d} \theta}_{:=\mathcal{J}} \\
& -\underbrace{\left.\int_{0}^{2 \pi} \frac{a(0, \theta)}{\partial_{r} \varphi(0, \theta)} \exp (\varphi(0, \theta) / h) \mathrm{d} \theta\right\} .}_{:=\mathcal{K}}
\end{aligned}
$$

On a $\mathcal{I}=O(1), \mathcal{J}=O(\exp (-\delta / h))$ et $\mathcal{K}=O(1)$ donc $I(h)=O(h)$. Comme $\mathcal{I}$ vérifie les mêmes hypothèses que $I(h)$ on a aussi $\mathcal{I}=O(h)$; d'où le résultat.

Proposition 6.3. - Il existe $r_{0}>0$ et des constantes $C, C^{\prime}>0$ tels que l'on ait

$$
\int_{\substack{0 \leq r \leq r_{0} \\ 0 \leq \theta<2 \pi}}(u \mid u) R(\theta) \mathrm{d} r \mathrm{~d} \theta \sim C h
$$

et pour $h$ appartenant $\grave{a}\left\{h_{k} ; k \in \mathbb{N}^{*}\right\}$

$$
\int_{\substack{0 \leq r \leq r_{0} \\ 0 \leq \theta<2 \pi}}\left(u^{+} \mid u^{-}\right) R(\theta) \mathrm{d} r \mathrm{~d} \theta=O\left(\exp \left(-C^{\prime} / h\right)\right) .
$$

De plus $u^{+}$et $u^{-}$sont linéairement indépendantes pour $h \in\left\{h_{k} ; k \in \mathbb{N}^{*}\right\}$ assez petit.

Démonstration. - Posons $P=\left[0, r_{0}\right] \times[0,2 \pi]$; on a

$$
\int_{P}(u \mid u) R(\theta) \mathrm{d} r \mathrm{~d} \theta=\int_{P} \sum_{\substack{1 \leq j \leq 2 \\ 1 \leq k \leq 2}}\left(u^{j} \mid u^{k}\right) \exp \left(i\left(\phi_{j}-\bar{\phi}_{k}\right) / h\right) R(\theta) \mathrm{d} r \mathrm{~d} \theta .
$$

Puisque $\partial_{r} \operatorname{Im} \phi_{j}(0, \theta)=\left(c_{R}^{-2}-c_{j}^{-2}\right)^{\frac{1}{2}}>0$, la régularité de $\operatorname{Im} \phi_{j}$ et les accroissements finis donnent l'existence de $r_{0}>0$ et $\delta>0$ tels que pour $\theta$ dans $[0,2 \pi]$,

$$
\forall r \in\left[0, r_{0}\right], \quad \operatorname{Im} \phi_{j}(0, \theta) \geq 0 \quad \text { et } \quad \forall r \in\left[\frac{r_{0}}{4}, r_{0}\right], \quad \operatorname{Im} \phi_{j}(0, \theta) \geq \delta .
$$

TOME $132-2004-\mathrm{N}^{\mathrm{O}} 2$ 
La propriété (39) reste valable pour tout $\theta$ dans $\mathbb{R}$ puisque $\operatorname{Im} \phi_{j}$ est $2 \pi$ périodique en la variable $\theta$ d'après la proposition 3.2 .

Posons $\varphi_{j k}=i\left(\phi_{j}-\bar{\phi}_{k}\right)$. On a $\varphi_{j k}=-\left(\operatorname{Im} \phi_{j}+\operatorname{Im} \phi_{k}\right)+i\left(\operatorname{Re} \phi_{j}-\operatorname{Re} \phi_{k}\right)$. D'après $(37), \varphi_{j k}(0, \theta)=0$. Dans $P, \operatorname{Re} \varphi_{j k}=-\left(\operatorname{Im} \phi_{1}+\operatorname{Im} \phi_{2}\right) \leq 0$ et $\operatorname{Re} \varphi_{j k}\left(r_{0}, \theta\right)<0$ d'après (39). De plus $\partial_{r} \operatorname{Re} \phi_{j}(0, \theta)=0$ donc $\partial_{r} \varphi_{j k}(0, \theta)=$ $-\left(c_{R}^{-2}-c_{j}^{-2}\right)^{\frac{1}{2}}-\left(c_{R}^{-2}-c_{k}^{-2}\right)^{\frac{1}{2}}$ et donc si $r_{0}$ est assez petit, on a $\partial_{r} \varphi_{j k} \neq 0$ dans $P$.

On peut donc appliquer le lemme 6.2 à chacun des quatres termes de (38) et on obtient

$$
\int_{P}(u \mid u) R(\theta) \mathrm{d} r \mathrm{~d} \theta=C h+O\left(h^{2}\right)
$$

avec

$$
C=\int_{0}^{2 \pi} \sum_{\substack{1 \leq j \leq 2 \\ 1 \leq k \leq 2}} \frac{1}{\alpha_{j}+\alpha_{k}}\left(u_{0}^{j}(0, \theta) \mid u_{0}^{k}(0, \theta)\right) R(\theta) \mathrm{d} \theta
$$

où l'on a posé $\alpha_{j}=\left(c_{R}^{-2}-c_{j}^{-2}\right)^{\frac{1}{2}}$.

L'inégalité de Cauchy-Schwarz pour le produit hermitien usuel (.|.) dans $\mathbb{C}^{2}$ donne

$$
\sum_{\substack{1 \leq j \leq 2 \\ 1 \leq k \leq 2}} \frac{1}{\alpha_{j}+\alpha_{k}}\left(u_{0}^{j}(0, \theta) \mid u_{0}^{k}(0, \theta)\right) \geq \frac{1}{2 \alpha_{1}}\left|u_{0}^{1}\right|^{2}+\frac{1}{2 \alpha_{2}}\left|u_{0}^{2}\right|^{2}-\frac{2}{\alpha_{1}+\alpha_{2}}\left|u_{0}^{1}\right| \cdot\left|u_{0}^{2}\right| .
$$

La forme quadratique réelle $q(x, y)=1 /\left(2 \alpha_{1}\right) x^{2}+1 /\left(2 \alpha_{2}\right) y^{2}-2 /\left(\alpha_{1}+\alpha_{2}\right) x y$ a pour mineurs principaux $1 /\left(2 \alpha_{1}\right)>0$ et $\left(\alpha_{1}-\alpha_{2}\right)^{2} /\left(4 \alpha_{1} \alpha_{2}\left(\alpha_{1}+\alpha_{2}\right)^{2}\right)>0$; elle est donc définie positive. Puisque $u_{0}^{1}(0, \theta)$ ne s'annule pas dans $[0,2 \pi]$ (voir la proposition 4.7 ), on a $C>0$.

Montrons maintenant la deuxième partie de la proposition. On a

$$
I:=\int_{P}\left(u^{+} \mid u^{-}\right) R(\theta) \mathrm{d} r \mathrm{~d} \theta=\int_{P} \sum_{\substack{1 \leq j \leq 2 \\ 1 \leq k \leq 2}}\left(u^{+j} \mid u^{-k}\right) \exp \left(i\left(\phi_{j}^{+}-\overline{\phi_{k}^{-}}\right) / h\right) R(\theta) \mathrm{d} r \mathrm{~d} \theta .
$$

Puisque la fonction sous le signe somme est analytique en la variable $\theta$ dans une bande complexe, on peut déformer le contour dans le complexe dans la variable $\theta$. Soit $y$ un nombre $>0$ qui sera choisi assez petit. On considère le contour $\gamma=\gamma_{1} \cup \gamma_{2} \cup \gamma_{3}$ où $\gamma_{1}$ est le segment de droite joignant 0 à $i y, \gamma_{2}$ est le segment de droite joignant $i y$ à $2 \pi+i y$ et $\gamma_{3}$ est le segment de droite joignant $2 \pi+i y$ à $2 \pi$. On a

$$
I=\int_{0}^{r_{0}}(\sum_{1 \leq \ell \leq 3} \underbrace{\int_{\gamma_{\ell}} u^{+}(r, \theta ; h) \cdot \overline{u^{-}(r, \bar{\theta} ; h)} R(\theta) \mathrm{d} \theta}_{:=I_{\ell}}) \mathrm{d} r .
$$

BULLETIN DE LA SOCIÉtÉ MATHÉMATIQUE DE FRANCE 
D'après (36) on a, pour $h$ dans $\left\{h_{k} ; k \in \mathbb{N}^{*}\right\}$,

$u^{ \pm}(r, \theta+2 \pi ; h)-u^{ \pm}(r, \theta ; h)=O\left(\exp \left(-\left(\min \left(\operatorname{Im} \phi_{1}^{ \pm}(r, \theta), \operatorname{Im} \phi_{2}^{ \pm}(r, \theta)\right)+C_{1}\right) / h\right)\right)$ où $C_{1}>0$. Donc d'après (39), si $y$ est assez petit, pour tout $r$ dans $\left[0, r_{0}\right]$ et tout $x$ dans $[0, y]$, on a

$$
u^{ \pm}(r, 2 \pi+i x ; h)-u^{ \pm}(r, i x ; h)=O\left(\exp \left(-C_{1} /(2 h)\right)\right)
$$

et donc aussi

$$
\overline{u^{ \pm}(r, 2 \pi-i x ; h)}-\overline{u^{ \pm}(r,-i x ; h)}=O\left(\exp \left(-C_{1} /(2 h)\right)\right) .
$$

On déduit de cela que

$$
I_{1}+I_{3}=O\left(\exp \left(-C_{1} /(2 h)\right)\right) .
$$

Il reste à estimer $I_{2}$. Posons $\varphi_{j k}=i\left(\phi_{j}^{+}-\overline{\phi_{k}^{-}}\right)$; on a

$$
\varphi_{j k}=-\operatorname{Im} \phi_{j}^{+}-\operatorname{Im} \phi_{k}^{-}+i\left(\operatorname{Re} \phi_{j}^{+}-\operatorname{Re} \phi_{k}^{-}\right) ;
$$

donc $\partial_{\theta} \varphi_{j k}(0, \theta)=2 i c_{R}^{-1} R(\theta)$. $\grave{A}$ une constante imaginaire pure près, on a

$$
\varphi_{j k}(0, \theta)=2 i c_{R}^{-1} \int_{\gamma^{\prime}} R(z) \mathrm{d} z
$$

où $\gamma^{\prime}$ est un chemin quelconque joignant 0 à $\theta$ pourvu que $\gamma^{\prime}$ soit contenu dans le domaine d'analyticité de $z \mapsto R(z)$. On va estimer $\operatorname{Re} \varphi_{j k}$ sur $\gamma_{2}$. Soit $s$ appartenant à $[0,2 \pi]$, on a

$$
\begin{aligned}
\operatorname{Re} \varphi_{j k}(0, s+i y) & =-2 c_{R}^{-1} \operatorname{Im}\left(\int_{0}^{s} R(x) \mathrm{d} x+\int_{0}^{y} R(s+i t) \mathrm{d}(s+i t)\right) \\
& =-2 c_{R}^{-1} \int_{0}^{y} \operatorname{Re} R(s+i t) \mathrm{d} t .
\end{aligned}
$$

Fixons $y>0$ assez petit tel que

$$
\forall s \in[0,2 \pi], \forall t \in[0, y], \quad \operatorname{Re} R(s+i t) \geq C_{2}>0 .
$$

On a alors $\operatorname{Re} \varphi_{j k}(0, s+i y) \leq-2 c_{R}^{-1} y C_{2}$. Si l'on choisit $r_{0}$ assez petit, on a

$$
\left.\operatorname{Re} \varphi_{j k}\right|_{\gamma_{2}} \leq-c_{R}^{-1} y C_{2}
$$

et donc

$$
I_{2}=O\left(\exp \left(-c_{R}^{-1} y C_{2} / h\right)\right) .
$$

Les estimations (40) et (41) donnent la deuxième partie de la proposition.

Supposons qu'il existe $\alpha^{+}(h)$ et $\alpha^{-}(h)$ tels que

$$
\alpha^{+}(h) u^{+}+\alpha^{-}(h) u^{-}=0 .
$$

TOME $132-2004-\mathrm{N}^{\mathrm{O}} 2$ 
On a alors

$$
\begin{aligned}
& \alpha^{+} \int_{P}\left(u^{+} \mid u^{+}\right) R(\theta) \mathrm{d} r \mathrm{~d} \theta+\alpha^{-} \int_{P}\left(u^{-} \mid u^{+}\right) R(\theta) \mathrm{d} r \mathrm{~d} \theta=0, \\
& \alpha^{+} \int_{P}\left(u^{+} \mid u^{-}\right) R(\theta) \mathrm{d} r \mathrm{~d} \theta+\alpha^{-} \int_{P}\left(u^{-} \mid u^{-}\right) R(\theta) \mathrm{d} r \mathrm{~d} \theta=0 .
\end{aligned}
$$

D'après les deux premières parties de la proposition, le déterminant de ce système d'inconnues $\alpha^{+}$et $\alpha^{-}$est équivalent à $C^{2} h^{2}$ donc est non nul pour $h$ assez petit. Il s'ensuit que $u^{+}$et $u^{-}$sont linéairement indépendantes.

Proposition 6.4. - Il existe deux fonctions $W^{+}(h)$ et $W^{-}(h), \mathcal{C}^{\infty}$ à support compact dans $\Omega$, de norme 1 dans $L^{2}(\Omega)$, et une constante $C>0$ telles que pour $h$ assez petit dans $\left\{h_{k} ; k \in \mathbb{N}^{*}\right\}$ on ait

$$
\begin{aligned}
& \left(-h^{2} \Delta_{e}-1\right) W^{ \pm}=O(\exp (-C / h)) \quad \text { dans } \Omega, \\
& B W^{ \pm}(h)=0 \quad \text { sur } \Gamma, \\
& \left(W^{+}(h), W^{-}(h)\right)_{L^{2}(\Omega)}=O(\exp (-C / h)) .
\end{aligned}
$$

En outre, $W^{+}(h)$ et $W^{-}(h)$ sont linéairement indépendantes.

Démonstration. - De la première partie de (39) on déduit avec (21) et (36) qu'il existe une constante $C_{1}>0$ telle que

$$
\begin{array}{rlrl}
Q(h) u & =O\left(\exp \left(-C_{1} / h\right)\right), & & 0<r \leq r_{0}, \theta \in \mathbb{R}, \\
B u & =O\left(\exp \left(-C_{1} / h\right)\right), \quad r=0, \theta \in \mathbb{R}
\end{array}
$$

et pour tout $h$ dans $\left\{h_{k} ; k \in \mathbb{N}^{*}\right\}$

$$
u(r, \theta+2 \pi ; h)-u(r, \theta ; h)=O\left(\exp \left(-C_{1} / h\right)\right) .
$$

Soit $\chi=\chi(r)$ une fonction $\mathcal{C}^{\infty}$ dans $[0,+\infty$ [ vérifiant $\chi(r)=1$ pour $0 \leq r \leq \frac{1}{4} r_{0}, \chi(r)=0$ pour $r \geq \frac{1}{2} r_{0}$ et $0 \leq \chi \leq 1$. On pose $v=\chi \cdot u$.

Pour $0<r<\frac{1}{4} r_{0}$, on a $\chi(r)=1$; donc $Q(h) v=Q(h) u$ et $B v_{\mid r=0}=B u_{\mid r=0}$. Pour $r>\frac{1}{2} r_{0}$, on a $v=0$; donc $Q(h) v=0$. Pour $\frac{1}{4} r_{0} \leq r \leq \frac{1}{2} r_{0}$, d'après la deuxième partie de $(39), u=O(\exp (-\delta / h))$. On obtient donc l'existence de $C_{2}=\min \left(C_{1}, \delta\right)>0$ telle que

$$
\begin{aligned}
Q(h) v & =O\left(\exp \left(-C_{2} / h\right)\right), & & r>0, \theta \in \mathbb{R}, \\
B v & =O\left(\exp \left(-C_{2} / h\right)\right), & & r=0, \theta \in \mathbb{R}, \\
v(r, \theta+2 \pi ; h)-v(r, \theta ; h) & =O\left(\exp \left(-C_{2} / h\right)\right), & & r \geq 0, \theta \in \mathbb{R},
\end{aligned}
$$

la dernière égalité ayant lieu pour $h \in\left\{h_{k} ; k \in \mathbb{N}^{*}\right\}$.

Posons $g(r, \theta ; h)=v(r, \theta+2 \pi ; h)-v(r, \theta ; h)$. La fonction $g=\left(\begin{array}{l}g_{1} \\ g_{2}\end{array}\right)$ est analytique en la variable $\theta$ dans une bande $|\operatorname{Im} \theta|<a$. On note alors $I g=\left(\begin{array}{l}I g_{1} \\ I g_{2}\end{array}\right)$ où

$$
\left(I g_{j}\right)(r, \theta ; h)=\frac{i}{4 \pi} \int_{\gamma} \operatorname{cotan}\left(\frac{1}{2}\left(\theta-\theta^{\prime}+0\right)\right) g_{j}\left(r, \theta^{\prime} ; h\right) \mathrm{d} \theta^{\prime}
$$

BULletin DE LA SOCIÉtÉ MATHÉMATIQUE DE FRANCE 
et on pose $w=v-I g$. On a

$w(r, \theta+2 \pi ; h)-w(r, \theta ; h)=g(r, \theta ; h)-((I g)(r, \theta+2 \pi ; h)-(I g)(r, \theta ; h))=0$.

D'après (36), on a

$$
u(r, \theta+2 \pi ; h)-u(r, \theta ; h)=O\left(\exp \left(-\left(\min \left(\operatorname{Im} \phi_{1}, \operatorname{Im} \phi_{2}\right)+C_{1}\right) / h\right)\right)
$$

pour $r$ dans un voisinage complexe $V$ de 0 et $\theta$ dans une bande complexe $B_{a}$. Si $V$ et $a>0$ sont assez petits, il existe donc une constante $C_{3}>0\left(C_{3}<C_{1}\right)$ telle que

$$
u(r, \theta+2 \pi ; h)-u(r, \theta ; h)=O\left(\exp \left(-C_{3} / h\right)\right)
$$

et d'après les inégalités de Cauchy, si $V$ et $a>0$ sont assez petits

(42) $\forall(r, \theta) \in V \times B_{a}, \partial_{r}^{\alpha} \partial_{\theta}^{\beta}(u(r, \theta+2 \pi ; h)-u(r, \theta ; h))=O\left(\exp \left(-C_{3} / h\right)\right)$.

Puisque $\chi$ est bornée indépendamment de $h$, d'après (42), si $0 \leq r \leq r_{0}$ et $|\operatorname{Im} \theta|<a$ avec $r_{0}$ et $a$ choisis assez petits alors $g=O\left(\exp \left(-C_{3} / h\right)\right)$. D'après le lemme 6.1 et les inégalités de Cauchy, on obtient alors

$$
\forall r \in\left[0, r_{0}\right], \forall \theta \in \mathbb{R}, \forall \alpha \in \mathbb{N}, \quad \partial_{\theta}^{\alpha}(I g)(r, \theta ; h)=O\left(\exp \left(-C_{3} / h\right)\right) .
$$

En dérivant sous le signe somme on obtient

$$
\partial_{r}^{\alpha}\left(I g_{j}\right)(r, \theta ; h)=\frac{i}{4 \pi} \int_{\gamma} \operatorname{cotan}\left(\frac{1}{2}\left(\theta-\theta^{\prime}+0\right)\right) \partial_{r}^{\alpha} g_{j}\left(r, \theta^{\prime} ; h\right) \mathrm{d} \theta^{\prime} .
$$

Puisque les dérivées de $\chi$ sont bornées indépendamment de $h$, d'après (42) on obtient pour $0 \leq r \leq r_{0}$ et $|\operatorname{Im} \theta|<a$

$$
\partial_{r}^{\alpha} g(r, \theta ; h)=O\left(\sum_{0 \leq k \leq \alpha} \partial_{r}^{k}(u(r, \theta+2 \pi ; h)-u(r, \theta ; h))\right)=O\left(\exp \left(-C_{3} / h\right)\right)
$$

et donc, de la même façon que pour le lemme 6.1, on obtient

$$
\forall r \in\left[0, r_{0}\right], \forall \theta \in \mathbb{R}, \forall \alpha \in \mathbb{N}, \quad \partial_{r}^{\alpha}\left(I g_{j}\right)(r, \theta ; h)=O\left(\exp \left(-C_{3} / h\right)\right) .
$$

D'après (43) et (44) on a

$$
Q(h) I g=O\left(\exp \left(-C_{3} / h\right)\right), \quad B I g_{\mid r=0}=O\left(\exp \left(-C_{3} / h\right)\right) .
$$

Il existe donc une constante $C_{4}=\min \left(C_{2}, C_{3}\right)>0$ telle que

$$
\begin{aligned}
Q(h) w & =O\left(\exp \left(-C_{4} / h\right)\right), \quad r>0, \theta \in \mathbb{R}, \\
B w & =O\left(\exp \left(-C_{4} / h\right)\right), \quad r=0, \theta \in \mathbb{R} .
\end{aligned}
$$

Posons $B w_{\mid r=0}=s$; on a la propriété classique suivante :

LEMME 6.5. - Il existe une fonction $w^{\prime}, \mathcal{C}^{\infty}$ à support compact dans la variable $r$ telle que

$$
B w_{\mid r=0}^{\prime}=s
$$

TOME $132-2004-\mathrm{N}^{\mathrm{O}} 2$ 
Preuve du lemme. - Cherchons $w^{\prime}$ telle que $w^{\prime}(0, \theta)=0$. On a $\partial_{\theta} w^{\prime}(0, \theta)=0$, donc $B w_{\mid r=0}^{\prime}=B_{0}(\theta) \partial_{r} w_{\mid r=0}^{\prime}$. Puisque $\operatorname{det} B_{0}(\theta)=\left(\lambda_{0}+\mu_{0}\right) \mu_{0} \neq 0, B_{0}(\theta)$ est inversible et l'équation (45) devient

$$
\partial_{r} w_{\mid r=0}^{\prime}=B_{0}(\theta)^{-1} s:=s^{\prime} .
$$

Il suffit alors de poser $w^{\prime}(r, \theta ; h)=\chi_{1}(r) r s^{\prime}(\theta ; h)$ où $\chi_{1}$ est une fonction $\mathcal{C}^{\infty}$ à support compact dans $\left[0,+\infty\left[\right.\right.$ valant 1 près de 0 . La fonction $w^{\prime}$ satisfait alors (46) et puisque $w^{\prime}(0, \theta)=0$, elle satisfait aussi(45).

Fin de preuve de la proposition 6.4. - On va estimer $\partial_{r}^{\alpha} \partial_{\theta}^{\beta} w^{\prime}$. On a

$$
\partial_{\theta}^{\beta} s=\left(\partial_{\theta}^{\beta} B w\right)_{\mid r=0}=O\left(\exp \left(-C_{4} / h\right)\right)
$$

et les dérivées par rapport à $r$ et $\theta$ de $\chi_{1}$ et $B_{0}(\theta)^{-1}$ sont bornées indépendamment de $h$; donc d'après la définition de $w^{\prime}$, on a $\partial_{r}^{\alpha} \partial_{\theta}^{\beta} w^{\prime}=$ $O\left(\exp \left(-C_{4} / h\right)\right)$. On a donc finalement en posant $W=w-w^{\prime}$

$$
\begin{aligned}
Q(h) W & =O\left(\exp \left(-C_{4} / h\right)\right), & & r>0, \theta \in \mathbb{R}, \\
B W & =0, & r & =0, \theta \in \mathbb{R},
\end{aligned}
$$

$w$ et $w^{\prime}$ étant à support compact en la variable $r, W$ l'est aussi.

La fonction $w$ étant $2 \pi$-périodique en la variable $\theta$, on obtient successivement que $s, s^{\prime}, w^{\prime}$ et donc $W$ le sont aussi. On peut donc identifier $W$ à une fonction $\mathcal{C}^{\infty}$ de la variable $x$ dans $\Omega$.

On a $W=u+O\left(\exp \left(-C_{4} / h\right)\right)$ et $u=O(1)$ d'après la première partie de $(39)$; donc

$$
\begin{aligned}
\|W\|_{L^{2}(\Omega)}^{2} & =\int_{\substack{0 \leq r \leq r_{0} \\
0 \leq \theta<2 \pi}} W \cdot \bar{W} R(\theta) \mathrm{d} r \mathrm{~d} \theta \\
& =\int_{\substack{0 \leq r \leq r_{0} \\
0 \leq \theta<2 \pi}} u \cdot \bar{u} R(\theta) \mathrm{d} r \mathrm{~d} \theta+O\left(\exp \left(-C_{4} / h\right)\right)=C h+O\left(h^{2}\right)
\end{aligned}
$$

et aussi

$$
\begin{aligned}
\left(W^{+}, W^{-}\right)_{L^{2}(\Omega)} & =\int_{\substack{0 \leq r \leq r_{0} \\
0 \leq \theta<2 \pi}} W^{+} \cdot \bar{W}^{-} R(\theta) \mathrm{d} r \mathrm{~d} \theta \\
& =\int_{\substack{0 \leq r \leq r_{0} \\
0 \leq \theta<2 \pi}} u^{+} \cdot \bar{u}^{-} R(\theta) \mathrm{d} r \mathrm{~d} \theta+O\left(\exp \left(-C_{4} / h\right)\right) \\
& =O\left(\exp \left(-\min \left(C^{\prime}, C_{4}\right) / h\right)\right) .
\end{aligned}
$$

Alors, de la même façon que pour $u^{+}$et $u^{-}$, de (47) et (48) on obtient que $W^{+}$ et $W^{-}$sont linéairement indépendants.

Et puisque $\|W\|_{L^{2}(\Omega)} \sim C^{\frac{1}{2}} h^{\frac{1}{2}}$ n'est pas exponentiellement petit, on obtient le résultat en renormalisant $W$.

BULletin DE LA SOCiÉtÉ MATHÉMATIQUE DE FRANCE 
6.3. Des quasimodes aux résonances de Rayleigh. - Nous sommes maintenant en mesure de prouver le théorème.

Les résultats de Stefanov [12] (voir le corollaire 1 et la remarque due à I. Nakamura) et la proposition 6.4 donnent l'existence d'au moins deux résonances (comptées avec multiplicités) exponentiellement proches de chacun des $\lambda_{k}:=h_{k}^{-1}$. On a donc la première partie du théorème.

Puisque $h_{k} \sim \ell(\Gamma) / 2 \pi c_{R} k^{-1}$ quand $k$ tend vers $+\infty$, on a

$$
\left|\operatorname{Re}\left(z_{k, \pm}\right)-\lambda_{k}\right|=O\left(\exp \left(-C \lambda_{k}\right)\right)=O\left(\exp \left(-C^{\prime} k\right)\right)
$$

où $C^{\prime}>0$, donc $\operatorname{Re}\left(z_{k, \pm}\right)$ a le même développement semi-classique de paramètre $k^{-1}$ que $\lambda_{k}=h_{k}{ }^{-1}$. Or, $h_{k}=\ell(\Gamma) /\left(2 \pi c_{R}\right) k^{-1}+\sum_{m \geq 2} e_{m} k^{-m}$ est un symbole analytique d'après la proposition 5.4 ; donc

$$
\lambda_{k}=\frac{2 \pi c_{R}}{\ell(\Gamma)} k+\sum_{m \geq 0} a_{m} k^{-m}
$$

est aussi un symbole analytique. $\operatorname{Re}\left(z_{k, \pm}\right)$ est donc un symbole analytique.

D'après Sjöstrand-Vodev [10], dans le cas d'un obstacle strictement convexe à bord $\mathcal{C}^{\infty}$ en dimension 2 , l'asymptotique du nombre de résonances de Rayleigh dans $\Lambda$ de module inférieur à $r$ est

$$
N(r)=\frac{\ell(\Gamma)}{\pi c_{R}} r+O(1), \quad r \longrightarrow+\infty .
$$

Soit $N_{q}(r)$ le nombre de $\lambda_{k}$ inférieur ou égal à $r$. D'après le développement de $\lambda_{k}$, on a

$$
\lambda_{k}=\frac{2 \pi c_{R}}{\ell(\Gamma)} k+a_{0}+O\left(\frac{1}{k}\right) .
$$

Donc pour $k$ assez grand $\left(\lambda_{k}\right)_{k \in \mathbb{N}}$ est une suite croissante et

$$
\lambda_{k-1}<\frac{2 \pi c_{R}}{\ell(\Gamma)} k+a_{0}<\lambda_{k+1}
$$

Soit $r$ assez grand; il existe un unique entier $k_{0}=\left[\ell(\Gamma) /\left(2 \pi c_{R}\right)\left(r-a_{0}\right)\right]$ vérifiant

$$
\frac{2 \pi c_{R}}{\ell(\Gamma)} k_{0}+a_{0}<r \leq \frac{2 \pi c_{R}}{\ell(\Gamma)}\left(k_{0}+1\right)+a_{0} .
$$

Donc d'après (49) appliqué à $k_{0}$ et $k_{0}+1$, on a $\lambda_{k_{0}-1}<r<\lambda_{k_{0}+2}$; donc

$$
k_{0}-1 \leq N_{q}(r) \leq k_{0}+1
$$

On en déduit

$$
\frac{\ell(\Gamma)}{2 \pi c_{R}}\left(r-a_{0}\right)-2<N_{q}(r) \leq \frac{\ell(\Gamma)}{2 \pi c_{R}}\left(r-a_{0}\right)+1
$$

et finalement

$$
N_{q}(r)=\frac{\ell(\Gamma)}{2 \pi c_{R}} r+O(1), \quad r \longrightarrow+\infty
$$

TOME $132-2004-\mathrm{N}^{\mathrm{O}} 2$ 
Pour les mêmes raisons, le nombre de résonances $z_{k, \pm}$ de module inférieur à $r$ est

$$
N_{ \pm}(r)=\frac{\ell(\Gamma)}{\pi c_{R}} r+O(1), \quad r \longrightarrow+\infty
$$

donc comme $z_{k, \pm}$ appartient à $\Lambda$, le nombre de résonances dans $\Lambda$ différentes des $z_{k, \pm}$ et de module inférieur à $r$ est $N(r)-N_{ \pm}(r)$ c'est-à-dire $O(1)$. Donc si $C_{0}$ est assez grand il n'y a pas d'autre résonance dans $\Lambda$ que les $z_{k, \pm}$.

\section{BIBLIOGRAPHIE}

[1] Bellassoued (M.) - Distributions of resonances and decay rate of the local energy for the elastic wave equation, Comm. Math. Phys., t. 215 (2000), pp. 375-408.

[2] Buslaev (V.) \& Fedotov (A.) - The complex WKB method for Harper's equation I, St. Petersburg Math. J., t. 6 (1995), pp. 495-517.

[3] Gamblin (D.) - Résonances de Rayleigh en dimension 2, Thèse, Université Paris 13, 2002.

[4] Grigis (A.) \& SuÖSTRAnd (J.) - Microlocal analysis for differential operators, An introduction, London Mathematical Society Lecture Note Series, vol. 196, Cambridge University Press, Cambridge, 1994.

[5] Helffer (B.) \& Sjöstrand (J.) - Analyse semi-classique pour l'équation de Harper II, Mém. Soc. Math. France, vol. 40, Société Mathématique de France, Paris, 1990.

[6] Ikehata (M.) \& Nakamura (G.) - Decaying and nondecaying properties of the local energy of an elastic wave outside an obstacle, Japan J. Appl. Math., t. 6 (1989), pp. 83-95.

[7] Kawashita (M.) - On the local-energy decay property for the elastic wave equation with the Neumann boundary conditions, Duke Math. J., t.67 (1992), pp. 333-351.

[8] NÉDÉLEC (L.) - Résonances semi-classiques pour l'opérateur de Schrödinger matriciel en dimension 2, Thèse, Université Paris 13, 1994.

[9] SjÖstrand (J.) - Singularités analytiques microlocales, Astérisque, vol. 95, Société Mathématique de France, Paris, 1982.

[10] Sjöstrand (J.) \& Vodev (G.) - Asymptotics of the number of Rayleigh resonances, Math. Ann., t. 309 (1997), pp. 287-306.

[11] Sjöstrand (J.) \& Zworski (M.) - Complex scaling method and the distribution of scattering poles, J. Amer. Math. Soc., t. 4 (1991), pp. 729-769.

[12] Stefanov (P.) - Quasimodes and resonances : Sharp lower bounds, Duke Math. J., t. 99 (1999), no. 1, pp. 75-92.

[13] L Lower bound of the number of the Rayleigh resonances for arbitrary body, Indiana Univ. Math. J., t. 49 (2000), no. 1, pp. 405-426.

BULletin DE LA SOCIÉtÉ MATHÉMATIQUE DE FRANCE 
[14] _ Resonance expansions and Rayleigh waves, Math. Res. Lett., t. 8 (2001), no. 1-2, pp. 105-124.

[15] Stefanov (P.) \& Vodev (G.) - Distribution of resonances for the Neumann problem in linear elasticity outside a ball, Ann. Inst. H. Poincaré (Phys. théor.), t. 60 (1994), no. 3, pp. 303-321.

[16] _ Distribution of resonances for the Neumann problem in linear elasticity in the exterior of a strictly convex body, Duke Math. J., t. 78 (1995), no. 3, pp. 677-714.

[17] _ Neumann resonances in linear elasticity for an arbitrary body, Comm. Math. Phys., t. 176 (1996), pp. 645-659.

[18] TAng (S.-H.) \& Zworski (M.) - From quasimodes to resonances, Math. Research Letters, t. 5 (1998), pp. 261-272.

[19] , Resonances expansions of scattered waves, Comm. Pure Appl. Math., t. 53 (2000), pp. 1305-1334.

[20] TAYLOR (M.) - Rayleigh waves in linear elasticity as a propagation of singularities phenomenon, in Partial differential equations and geometry (Proc. Conf., Park City, Utah, 1977), Lecture Notes in Pure and Appl. Math., vol. 48, Marcel Dekker, New York, 1979, pp. 273-291.

[21] Vodev (G.) - Existence of Rayleigh resonances exponentially close to the real axis, Ann. Inst. H. Poincaré (Phys. théor.), t. 67 (1997), no. 1, pp. 4157. 